\title{
Linear stability analysis of travelling waves for a pseudo-parabolic Burgers' equation
}

\author{
C. M. Cuesta
}

ABSTRACT. We investigate the linear stability of non-monotone travelling wave solutions of the pseudo-parabolic Burgers' type equation

$$
\frac{\partial u}{\partial t}=\frac{\partial^{2} u}{\partial x^{2}}+2 u \frac{\partial u}{\partial x}+\tau \frac{\partial^{2} u}{\partial x^{2} \partial t} \quad \text { with } \quad \tau>0
$$

The monotonicity of the waves depending on the strength of the parameter $\tau$ and the far-filed values. The most part of the paper is devoted to prove that the linear stability is determined by the spectrum of the linearised operator. This step is necessary since the linearised operator does not fall into the class of sectorial operators, for which the result is well-known. An Evans function is defined and used to search for instabilities numerically. The numerical results yield the conclusion that no eigenvalues with positive real part appear and hence that stability of the waves holds.

\section{CONTENTS}

1. Introduction $\quad 77$

2. The linearised operator and the Evans function 82

3. Large $|\lambda| \quad 88$

4. Analysis of the resolvent kernel 93

5. Remarks on asymptotic stability on weighted spaces 96

6. Large $\tau$ : numerical search for zeros $\quad 98$

$\begin{array}{lr}\text { Appendix } & 100\end{array}$

$\begin{array}{ll}\text { References } & 104\end{array}$

\section{Introduction}

We analyse the linear stability of travelling wave solutions to the problem

$$
\frac{\partial u}{\partial t}=\frac{\partial^{2} u}{\partial x^{2}}+2 u \frac{\partial u}{\partial x}+\tau \frac{\partial^{3} u}{\partial x^{2} \partial t} \quad \text { in } \quad \mathbb{R} \times \mathbb{R}^{+},
$$

1991 Mathematics Subject Classification. Primary 35B40, 35B35; Secondary 35G20 .

Key words and phrases. pseudo-parabolic equations, travelling waves, Evans function.

The author was supported by an EPSRC Postdoctoral Research Fellowship while at the University of Nottingham. 
with initial condition

$$
u(x, 0)=u_{0}(x) \quad \text { in } \quad \mathbb{R}
$$

where, for definiteness, $u_{0}$ is a bounded continuous function and satisfies

$$
u_{0}(-\infty)=0, \quad u_{0}(+\infty)=1 .
$$

Equation (1.1) was introduced as a pilot-problem of the model of unsaturated groundwater flow presented in [6]. Equation (1.1) is also referred in the literature as the Benjamin-Bona-Mahony-Burgers' (BBM-Burgers) equation, or a viscous regularisation of the original $\mathrm{BBM}$ model for long wave propagation, see [2].

The travelling wave solutions connecting $\phi(-\infty)=0$ to $\phi(+\infty)=1$ are unique up to translation and correspond to a unique orbit connecting the saddle $(0,0)$ to the sink $(1,1)$ in the phase plane defined by the pair $(\phi, \psi)$ where $\psi=\phi+\tau \phi^{\prime}$. Thus, the unique orbit coming out of $(0,0)$ into the first quadrant connects to $(1,1)$. This follows from a standard argument that relies, in particular, on the negativity of the divergence of the vector field. It is easy to see, by linearisation about $(1,1)$, that $\phi$ is monotone increasing if $\tau \leq \frac{1}{4}$.

Nonlinear stability of travelling wave solutions is partially answered in [6]: energy estimates are here used to conclude the result, however they only apply to monotone travelling wave solutions, in particular, to the cases with $\tau \leq \frac{1}{4}$. This parameter has the effect of increasing the number of oscillations around the state $\phi \equiv 1$ as it gets larger. The question we address here is then whether travelling wave solutions are stable for $\tau>\frac{1}{4}$. The second purpose of this paper is to establish a basis for the study of linear stability in more complex pseudo-parabolic systems, such us models of two-phase porous media flow that include a dynamic pressure relation as postulated in $[\mathbf{1 1}]$. For a formulation and analysis of the travelling wave equation in the case of unsaturated flow under the action of gravity, see [7].

We first give an account of the basic ideas, as well as address some difficulties of the method and the relevant literature. Section 2 is devoted to setting up the problem, it provides a preliminary analysis of the linearised operator and its eigenvalue problem. We prove that this operator generates a $C_{0}$-semigroup. Next we locate the essential spectrum and define the Evans function, $D(\lambda)$ with $\lambda$ in a suitable domain $\Omega \subset \mathbb{C}$. The zeros of $D(\lambda)$ are eigenvalues of the linearised operator, and so it gives us a way of studying the spectrum of the linearised operator that is exploited later.

In Section 3 the limit $D(\lambda) \rightarrow 1$ as $|\lambda| \rightarrow \infty$ is proved for $\tau>1 / 4$ fixed. This result and the continuity of the Evans function in $\tau$ help us carry out a numerical computation to find eigenvalues. In Section 6 we described the numerical method and give examples for different values of $\tau$. The examples lead to the conclusion that no eigenvalues with positive real part appear for large values of $\tau$, and hence stability of travelling wave solutions is expected. As we shall discuss below, in order to conclude stability we in addition have to prove that the upper bound of the spectrum of the linearised operator actually coincides with the growth abscissa of the semigroup generated by it. This is done in Section 4 . We use a result by Prüss [23], by which we only need to prove that for every $\delta>0$ the resolvent operator is uniformly bounded in $\lambda$ for $\operatorname{Re}(\lambda) \geq \delta$. The necessary estimates are achieved by using a Green's function formulation of the resolvent. Finally, we have used exponential dichotomies in order to construct the Green's function. These are generalisation of the unstable and stable manifolds for systems of linear ODEs 
with constant coefficients. This framework has been well established in [5], [18] and $[\mathbf{1 9}]$, and account of the results we apply here can be found in the Appendix.

Preliminaries. First we give a brief overview of the general method. Let us consider a general initial value problem

$$
\begin{aligned}
& \frac{\partial u}{\partial t}=B u+F\left(u, \frac{\partial u}{\partial x}\right) \quad \text { on } \mathbb{R} \times[0, T] \\
& u(0, x)=u_{0}(x) \quad \text { in } \mathbb{R}
\end{aligned}
$$

where $B$ is a linear operator, and $F(U, V)$ is nonlinear. We assume that problem (1.3) is well-posed in a Banach space $X$, which is typically $L^{\infty}(\mathbb{R})$, or the space of uniformly continuous functions. We also assume that (1.3) admits travelling wave solutions, i.e solutions of the form $u(x, t)=\phi(x+c t)$, with $c>0$, that connect two constant states: $\phi(+\infty)=\phi^{+}$to $\phi(-\infty)=\phi^{-}$.

Before performing the linearisation it is convenient to transform the equation to the travelling wave coordinate $\eta=x+c t$, so that a travelling wave $\phi(\eta)$ is a stationary solution of the resulting equation. Let $z:=u-\phi$ and (1.3) becomes

$$
\frac{\partial z}{\partial t}+c \frac{\partial z}{\partial \eta}=B z+B \phi+F\left(z+\phi, \frac{\partial z}{\partial \eta}+\phi^{\prime}\right)-c \phi^{\prime}
$$

Since $\phi$ satisfies the equation

$$
c \phi^{\prime}=B \phi+F\left(\phi, \phi^{\prime}\right)
$$

the linear part of (1.4) reads

$$
\frac{\partial z}{\partial t}=B z+\frac{\partial F\left(\phi, \phi^{\prime}\right)}{\partial U} z+\frac{\partial F\left(\phi, \phi^{\prime}\right)}{\partial V} \frac{\partial z}{\partial \eta}-c \frac{\partial z}{\partial \eta}:=L z .
$$

We further assume that the operator $L$ is an infinitesimal generator of a $C_{0^{-}}$ semigroup $T(t)$. Then an estimate of the form

$$
\left\|T(t) z_{0}\right\|<M e^{\omega t} \quad \text { for } t \geq 0
$$

holds for the solution $z(t)=T(t) z_{0}$ of (1.5), see [20]. The infimum of all possible $\omega$ 's such that (1.6) holds is called the type or growth abscissa of the semigroup $T(t)$. Clearly if this is negative, travelling wave solutions are asymptotically linearly stable, in the sense that $\|z\| \rightarrow 0$ as $t \rightarrow \infty$, where $z$ is a solution of the linearised equation (1.5), and if is 0 stability holds. If further the nonlinear operator is locally Lipschitz continuous in $X$ then linear stability implies nonlinear stability.

The classical method of finding the type of the semigroup relies on the fact that for most of these problems, the linearised operator $L$ is sectorial and hence it generates an analytic semigroup $T(t)$, see $[\mathbf{2 0}]$, or equivalently there exist $a \in \mathbb{R}$, $\theta \in\left(\frac{\pi}{2}, \pi\right)$ and $M \geq 0$, such that the sector of the complex plane

$$
\mathcal{S}:=\{\lambda \in \mathbb{C}: 0 \leq|\arg (\lambda-a)| \leq \theta, \lambda \neq a\}
$$

is contained in the resolvent set of $L$, and the resolvent operator satisfies the estimate

$$
\left\|(\lambda I-L)^{-1}\right\| \leq \frac{M}{|\lambda-a|} \quad \text { on } \mathcal{S} .
$$

The last estimate allows to get the following representation of the semigroup

$$
T(t)=\frac{1}{2 \pi i} \int_{\Gamma}(\lambda I-L)^{-1} e^{\lambda t} d \lambda,
$$


where $\Gamma$ is a contour of the spectrum $\sigma(L)$ of $L$. The contour $\Gamma$ can be taken with $\arg \lambda \rightarrow \pm \theta$ as $|\lambda| \rightarrow \infty$ for some $\theta \in\left(\frac{\pi}{2}, \pi\right)$, which together with the estimate (1.7) implies that the integral (1.8) exists. As a consequence of (1.8) and the semigroup being analytic, the following form of the spectral mapping theorem holds:

$$
e^{\sigma(L) t}=\sigma(T(t)) \backslash\{0\} \quad \text { for all } t \geq 0 .
$$

The proof can be found in $[\mathbf{1 6}]$.

In general if the spectral mapping theorem (1.9) holds for a semigroup and its infinitesimal generator, then the type of the semigroup coincides with the spectral bound of $L$, i.e. with $\sup \{\operatorname{Re}(\lambda): \lambda \in \sigma(L)\}$. The analysis of the stability for the zero solution of the linearised problem (1.5) then reduces to finding the sign of the spectral bound of $L$.

We also observe that if $L$ generates a $C_{0}$-semigroup, the spectral inclusion

$$
e^{\sigma(L) t} \subset \sigma(T(t)), \quad \text { for } t \geq 0,
$$

holds, and therefore the type of the semigroup is always greater than or equal to the spectral bound of $L$. In particular, the spectral bound of $L$ being positive is a sufficient condition for (linear) instability.

The first difficulty in the application of these ideas to our problem, is the fact that the linearised operator for equation (1.1) does not generate an analytic semigroup. Moreover, it is not known whether the spectral mapping theorem holds in this case. However, we will be able to prove that the type and the spectral bound for our problem coincide, or that the spectrum determined growth property (SDG) holds. Indeed, in our case, from Corollary 3 in [23], a necessary and sufficient condition for the SDG property to hold is that for all $\delta>0$ the resolvent operator $(\lambda I-L)^{-1}$ is uniformly bounded in $\{\lambda \in \mathbb{C}: \operatorname{Re}(\lambda) \geq \delta\}$ provided that $\lambda=0$ is the eigenvalue with the largest real part. To tackle the estimates we shall write the resolvent operator as

$$
(L-\lambda I)^{-1} f(\eta)=\int_{\mathbb{R}} G_{\lambda}(\eta, \xi) f(\xi) d \xi \quad \eta \in \mathbb{R}
$$

where $G_{\lambda}$ is the Green's function associated to the equation $(L-\lambda I) u=f$ for a given $f$, i.e. $G_{\lambda}(\cdot, \xi)=(I-\lambda I)^{-1} \delta_{\xi}$ where $\delta_{\xi}$ is the delta function with point mass $\xi$. We then obtain uniform estimates on the resolvent kernel $G_{\lambda}$. The result in $[\mathbf{2 3}]$ holds in a Hilbert space, for that reason we will be working on $H^{2}$ and use that following inequality:

$$
\left\|\int_{\mathbb{R}} G_{\lambda}(\eta, \xi) f(\xi) d \xi\right\|_{L^{2}} \leq\left(\sup _{\xi \in \mathbb{R}}\left\|G_{\lambda}(\cdot, \xi)\right\|_{L_{\eta}^{1}(\mathbb{R})}\right)^{\frac{1}{2}}\left(\sup _{\eta \in \mathbb{R}}\left\|G_{\lambda}(\eta, \cdot)\right\|_{L_{\xi}^{1}(\mathbb{R})}\right)^{\frac{1}{2}}\|f\|_{L^{2}(\mathbb{R})} .
$$

The proof of (1.10) is analogous to that for the convolution product: $\|f * g\|_{L^{p}} \leq$ $\|f\|_{L^{1}}\|g\|_{L^{p}}$ (Young-Hausdorff inequality), see e.g. $[3]^{1}$. Essentially the main result of this paper is that for the linearised operator about a travelling waves solution

is

\footnotetext{
${ }^{1}$ The inequality (1.10) for a general $p \geq 1$ (including $p=\infty$ ), with $p^{\prime}$ such that $1 / p+1 / p^{\prime}=1$,
}

$$
\left\|\int_{\mathbb{R}} G_{\lambda}(\eta, \xi) f(\xi) d \xi\right\|_{L^{p}} \leq\left(\sup _{\xi \in \mathbb{R}}\left\|G_{\lambda}(\cdot, \xi)\right\|_{L_{\eta}^{1}}\right)^{\frac{1}{p}}\left(\sup _{\eta \in \mathbb{R}}\left\|G_{\lambda}(\eta, \cdot)\right\|_{L_{\xi}^{1}}\right)^{\frac{1}{p^{\prime}}}\|f\|_{L^{p}}
$$


associated to (1.1) then $\sup _{\xi \in \mathbb{R}}\left\|G_{\lambda}(\cdot, \xi)\right\|_{L_{\eta}^{1}(\mathbb{R})}<\infty$ and $\sup _{\xi \in \mathbb{R}}\left\|G_{\lambda}(\eta, \cdot)\right\|_{L_{\xi}^{1}(\mathbb{R})}<$ $\infty$ uniformly in $\lambda \in \rho(L)$.

Howard and Zumbrun in [14] concluded stability results of dispersive-diffusive waves for KdV type of equations by first estimating the resolvent kernel of the linearised operator. These estimates are later used to prove that an evolutionary Green's function of the form (1.8) can be constructed. Further estimates on the Green's function then give the stability (with long time decay rates) and instability results. Such ideas were first applied for viscous shocks waves in [13] and [27]. We postpone a similar investigation in our case, however, setting here the basis of such, more intricate, analysis.

The first step of the analysis is to locate the essential spectrum, i.e. the spectrum of $L$ aside from isolated eigenvalues with finite multiplicity. Even though in most cases the essential spectrum is contained in the left half plane, instability can still originate from the appearance of isolated eigenvalues in the right half plane; so after locating the essential spectrum it is necessary to look at the eigenvalue problem

$$
(L-\lambda I) \Psi=0 .
$$

In $L^{p}$ or more regular spaces the eigenvalues are those values of $\lambda$ for which there exist non-trivial bounded solutions of (1.11), see [12]. In order to find eigenvalues equation (1.11) is written as a system of first order linear ODEs

$$
Y^{\prime}=A(\lambda, \eta) Y
$$

where $A(\lambda, \eta)$ is an $n \times n$ matrix, $n$ being the order of equation (1.5), and $Y$ is the column vector $\left(\Psi, \Psi^{\prime} \ldots \Psi^{(n)}\right)$.

The coefficients of $A(\eta, \lambda)$ depend on $\phi$ and $\phi^{\prime}$. This implies that the matrix $A(\lambda, \eta)$ tends to constant matrices $A^{ \pm}(\lambda)$ as $\eta \rightarrow \pm \infty$. By standard results on asymptotic behaviour of ordinary differential equations, see [4], solutions of (1.5) behave as solutions of the constant coefficient equations $Y^{\prime}=A^{ \pm} Y$ as $\eta$ approaches $\pm \infty$, hence bounded solutions of (1.12) must decay exponentially to 0 at both $\eta= \pm \infty$. This can be measured in terms of a vanishing determinant of a set of solutions of (1.12). If $\lambda$ is away from the essential spectrum, then a set of solutions of (1.12) can be formed by $k$ independent solutions that decay to 0 at $\eta=-\infty$, and $n-k$ independent solutions that decay to 0 as $\eta=\infty$. When the determinant (or Wronskian) of this set is zero, a linear combination of these solutions give a bounded solution, hence $\lambda$ is an eigenvalue. This determinant is the so-called Evans function, see $[\mathbf{1 5}],[\mathbf{1}]$ and $[\mathbf{2 1}]$ for more precise definitions. Thus the Evans function has the properties of being analytic in $\lambda$ aside from the essential spectrum, and its zeros on this domain are isolated eigenvalues of $L$.

Finally, observe that $\lambda=0$ is always an eigenvalue since translation invariance of the equation (1.5) implies $L \phi^{\prime}=0$. If $\lambda=0$ is isolated, and the rest of the spectrum lies in the left half plane one can consider the projection on $X_{1}:=N(L)=$ $\operatorname{span}\left(\phi^{\prime}\right)^{2}$, so that $X=X_{1} \oplus X_{2}$. This allows to pose the linear problem in $X_{2}$, where the spectral bound is strictly negative. An estimate of the form (1.6) holds on $X_{2}$ and hence linear asymptotic stability also holds, see [9] or [12]. If zero is contained in the essential spectrum, a weighted norm might be introduced in a way that the spectrum of the operator in the weighted space is pushed off the imaginary

\footnotetext{
${ }^{2}$ Here $N(L)$ denotes the null space of $L$.
} 
axis, leaving the zero eigenvalue isolated. A typical example in which stability is studied in weighted spaces is the diffusive Burgers' equation, see [12] and [26].

\section{The linearised operator and the Evans function}

In this section we formulate the eigenvalue problem for the linearised operator $L$ resulting from equation (1.1). We first prove that the linearised operator generates a $C^{0}$-semigroup. Next we locate the essential spectrum and define the Evans function. We finish the section by proving that the eigenvalue $\lambda=0$ is a simple zero of the Evans function.

We let $\phi$ denote a travelling wave solution of (1.1). Then substituting $u(x, t)=$ $\phi(x+c t)$, we have for $\phi(\eta)$ with $\eta:=x+c t$, restricting attention to waves such that $\phi(-\infty)=0$ and $\phi(+\infty)=1$ and after integration with respect to $\eta$,

$$
\tau \phi^{\prime \prime}+\phi^{\prime}+\phi^{2}-\phi=0,
$$

here $c=1$ by the Rankine-Hugoniot condition (obtained by integrating in $\eta$ over the whole $\mathbb{R}$ ). Linearisation around a travelling wave solution leads for $U=u-\phi$ to the linear operator

$$
L U:=\left(I-\tau \frac{\partial^{2}}{\partial \eta^{2}}\right)^{-1}\left(\frac{\partial^{2} U}{\partial \eta^{2}}+2 \frac{\partial}{\partial \eta}(\phi U)\right)-\frac{\partial U}{\partial \eta} .
$$

By adopting the notation

$$
A_{\tau} u:=\left(I-\tau \frac{\partial^{2}}{\partial \eta^{2}}\right)^{-1} \frac{\partial^{2} u}{\partial \eta^{2}}, \quad B_{\tau} u:=\left(I-\tau \frac{\partial^{2}}{\partial \eta^{2}}\right)^{-1} \frac{\partial u}{\partial \eta},
$$

the linearised operator reads

$$
L U=A_{\tau} U+2 B_{\tau}(\phi U)-\frac{\partial U}{\partial \eta} .
$$

With this formulation the next proposition follows easily.

Proposition 2.1. The operator $L$ generates a $C_{0}$-semigroup in $H^{m}(\mathbb{R})$ with $m \geq 0$.

Proof. First observe that $A_{\tau}$ is a bounded operator in $L^{2}(\mathbb{R})$ (see e.g. [6]), and so it is also continuous in $H^{m}(\mathbb{R})$. Therefore it generates a $C_{0}$-semigroup, that we call $S(t)$. If $T_{a}$ denotes the translation operator

$$
T_{a}(u(\eta))=u(\eta-a),
$$

then for all $t \in \mathbb{R}, T_{t}$ is the group generated by the operator $U \rightarrow-\partial U / \partial \eta$. An easy computation shows that $A_{\tau}-\frac{\partial}{\partial \eta}$ generates the semigroup $T_{t} S(t)$. This is a

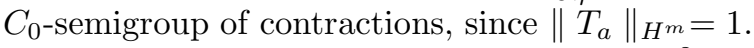

The operator $U \rightarrow 2 B_{\tau} U$ is bounded in $L^{2}(\mathbb{R})$ (see $[6]$ ) and it is in $H^{m}(\mathbb{R})$. This and the fact that $\phi$ and $\phi^{\prime}$ are bounded imply that the operator $U \rightarrow 2 B_{\tau}(\phi U)$ is bounded in $H^{m}(\mathbb{R})$ as well. Hence the operator $U \rightarrow L U=A_{\tau} U-\partial U / \partial \eta+2 B_{\tau} \phi U$ generates a $C_{0}$-semigroup in $H^{m}(\mathbb{R})$, see $[\mathbf{2 0}]$.

Before we study the eigenvalue problem we write the operator as

$$
L-\lambda I \equiv\left(I-\tau \frac{\partial^{2}}{\partial \eta^{2}}\right)^{-1}\left(\tilde{L}-\lambda\left(I-\tau \frac{\partial^{2}}{\partial \eta^{2}}\right)\right),
$$


with

$$
\tilde{L} u=\frac{\partial^{2} u}{\partial \eta^{2}}+2 \frac{\partial \phi u}{\partial \eta}-\left(\frac{\partial u}{\partial \eta}-\tau \frac{\partial^{3} u}{\partial \eta^{3}}\right) .
$$

The operator $\left(I-\tau \partial^{2} / \partial \eta^{2}\right)^{-1}$ is continuous from $H^{m}(\mathbb{R})$ to $H^{m+2}(\mathbb{R})$ (see e.g. [3]) for $m \geq 0$ and has

$$
\left\|I-\tau \frac{d^{2}}{d \eta^{2}}\right\|_{B\left(H^{m}(\mathbb{R}), H^{m+2}(\mathbb{R})\right)} \leq C_{\tau} \quad \text { with } C_{\tau}= \begin{cases}\tau & \text { if } \tau>1 \\ 1 & \text { if } \tau \leq 1\end{cases}
$$

The operators $\left(\tilde{L}-\lambda\left(I-\tau \partial^{2} / \partial \eta^{2}\right)\right)$ are continuous from $H^{m+2}(\mathbb{R})$ to $H^{m}(\mathbb{R})$. We take $m=0$, this is the case of minimal regularity tractable with our approach (see below). Thus we study the spectrum of $L$ in $H^{2}(\mathbb{R})$, and this is equivalent to studying the generalised eigenvalue problem

$$
\left(\tilde{L}-\lambda\left(I-\tau \frac{\partial^{2}}{\partial \eta^{2}}\right)\right) U=0 \quad \text { for } \quad U \in H^{2}(\mathbb{R})
$$

It is straightforward to prove that $\lambda$ is in the resolvent set of $L$ if, and only if,

$$
\left(\tilde{L}-\lambda\left(I-\tau \frac{\partial^{2}}{\partial \eta^{2}}\right)\right)^{-1}: L^{2}(\mathbb{R}) \rightarrow H^{2}(\mathbb{R})
$$

is continuous, and that $\lambda$ is in the point spectrum of $L$ if, and only if $(\tilde{L}-\lambda(I-$ $\left.\tau \partial^{2} / \partial \eta^{2}\right)$ ) is Fredholm of index zero as an operator from $H^{2}(\mathbb{R})$ to $L^{2}(\mathbb{R})$. In all other cases $\lambda$ belongs to the essential spectrum of $L$.

The minimal regularity required allows to study (2.4) as a system of linear ODEs. Letting $Y:=\left(U, U^{\prime}, U^{\prime \prime}\right)$, then $(2.4)$ reads

$$
\frac{d Y}{d \eta}=A(\eta, \lambda) Y
$$

with

$$
A(\eta, \lambda)=\left(\begin{array}{ccc}
0 & 1 & 0 \\
0 & 0 & 1 \\
\frac{\lambda-2 \phi^{\prime}(\eta)}{\tau} & \frac{1-2 \phi(\eta)}{\tau} & -\lambda-\frac{1}{\tau}
\end{array}\right)
$$

The following properties are satisfied by the matrix $A(\eta, \lambda)$ :

$\mathbf{P 1} A(\eta, \lambda)$ is analytic with respect to $\lambda$ for every $\eta \in \mathbb{R}$ and $\tau>0$ fixed.

P2 The asymptotic matrices $A^{ \pm}(\lambda):=\lim _{\eta \rightarrow \pm \infty} A(\eta, \lambda)$ are well defined. The convergence is uniform in $\lambda$.

Moreover, there exist $\alpha^{+}, \alpha^{-}>0$ such that

$$
\begin{gathered}
\left\|A(\eta, \lambda)-A^{+}(\lambda)\right\| \leq C e^{-\alpha^{+} \eta} \quad \text { for } \eta \geq 0, \\
\left\|A(\eta, \lambda)-A^{-}(\lambda)\right\| \leq C e^{+\alpha^{-} \eta} \quad \text { for } \eta \leq 0
\end{gathered}
$$

where the constants $C$ depend on $\tau$.

The property P2 follows from the exponential decay of $\phi$ and $\phi^{\prime}$ as $|\eta| \rightarrow$ $+\infty$. We recall that $\phi$ corresponds to a unique orbit in the phase plane connecting a saddle point to a sink. The exponents $\alpha^{+}$and $\alpha^{-}$giving the decay of $\phi$ as $\eta \rightarrow+\infty$ and $\eta \rightarrow-\infty$ respectively. In our case, $\alpha^{-}=(\sqrt{1+4 \tau}-1) / 2 \tau$ and $\alpha^{+}=\operatorname{Re}(1+\sqrt{1-4 \tau}) /(2 \tau)$ or $\operatorname{Re}(1-\sqrt{1-4 \tau}) /(2 \tau)$ depending on whether the orbit enters the sink through the slow (as is the case for monotone waves) or the fast direction (see $[6]$ ). But, obviously, $\alpha^{+}=1 /(2 \tau)$ for $\tau>1 / 4$. 
The characteristic polynomials corresponding to the asymptotic matrices read

$$
\begin{gathered}
P_{\lambda}^{+}(\mu)=\tau \mu^{3}+(1+\tau \lambda) \mu^{2}+\mu-\lambda \text { for } A^{+}(\lambda), \\
P_{\lambda}^{-}(\mu)=\tau \mu^{3}+(1+\tau \lambda) \mu^{2}-\mu-\lambda \text { for } A^{-}(\lambda) .
\end{gathered}
$$

In order to study the spectrum of $L$, we have to understand in which regions of $\mathbb{C}$ it is possible to construct bounded solutions of (1.11), i.e. for which values of $\lambda \mathrm{a}$ matching of solutions decaying to 0 at $-\infty$ with solutions that decay to 0 at $+\infty$ is possible. We look at the roots of the characteristic polynomials $P_{\lambda}^{ \pm}$. First we introduce the necessary notation.

Each polynomial $P_{\lambda}^{ \pm}$has three complex roots (counting multiplicity) for every fixed $\lambda$ in $\mathbb{C} . A^{ \pm}(\lambda)$ being analytic in $\lambda$ implies that the number of eigenvalues (counting multiplicity) of $A^{ \pm}(\lambda)$ having negative (resp. positive) real part is constant as $\lambda$ varies inside any of the connected components of the sets $\mathbb{C} \backslash S^{+}$and $\mathbb{C} \backslash S^{-}$, where

$$
S^{ \pm}:=\left\{\lambda \in \mathbb{C}: A^{ \pm}(\lambda) \text { has purely imaginary eigenvalues }\right\} .
$$

Those sets for $A^{ \pm}$are

$$
\begin{aligned}
& S^{+}=\left\{\lambda \in \mathbb{C}: \operatorname{Re}(\lambda)=\frac{-s^{2}}{1+\tau s^{2}}, \operatorname{Im}(\lambda)=\frac{s\left(1-\tau s^{2}\right)}{1+\tau s^{2}}, s \in \mathbb{R}\right\} \\
& S^{-}=\left\{\lambda \in \mathbb{C}: \operatorname{Re}(\lambda)=\frac{-s^{2}}{1+\tau s^{2}}, \operatorname{Im}(\lambda)=-s, s \in \mathbb{R}\right\}
\end{aligned}
$$

see also Figure 1. It is easily seen that $S^{+}$and $S^{-}$only intersect at $\lambda=0$ (at $s=0$ in the above parameterisations), and that otherwise $S^{-}$is to the right of $S^{+}$in $\mathbb{C}$. The simply connected components of $\mathbb{C} \backslash S^{+}$are $\Omega_{r}^{+}$, which is the component

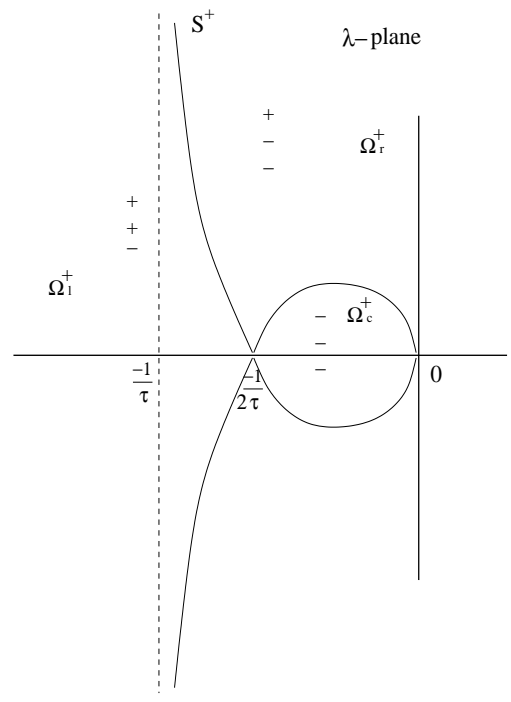

(a) Sign of real part of $\mu^{+'}$ s.

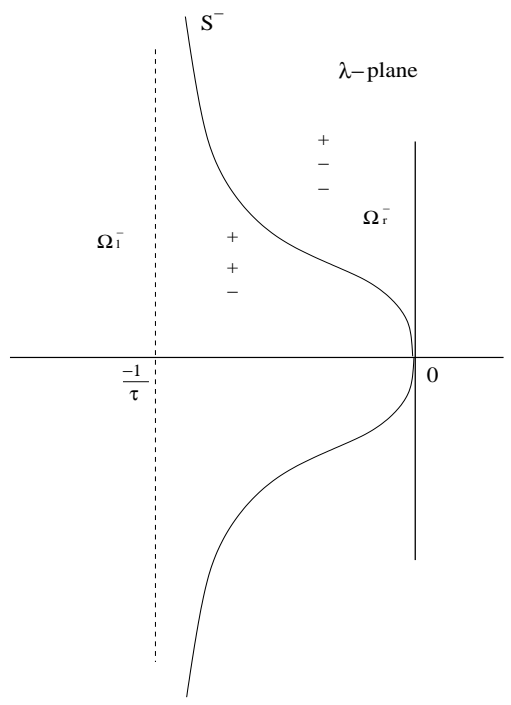

(b) Sign of real part of $\mu^{-}$s.

Figure 1. Distribution of sign of eigenvalues of $A^{+}$and $A^{-}$.

containing the interval $(0,+\infty), \Omega_{c}^{+}$, the one containing the interval $\left(-\frac{1}{2 \tau}, 0\right)$, and $\Omega_{l}^{+}$, which contains the interval $\left(-\infty,-\frac{1}{2 \tau}\right)$. The simply connected components of 
$\mathbb{C} \backslash S^{-}$are: $\Omega_{r}^{-}$, containing the interval $(0,+\infty)$, and $\Omega_{l}^{+}$, containing the interval $(-\infty, 0)$.

In the sequel, $\mu_{i}^{ \pm}(\lambda)$ for $i \in\{1,2,3\}$ will denote the eigenvalues of $A^{ \pm}(\lambda)$. And in the $\lambda$-regions where $A^{+}$and $A^{-}$have three distinct eigenvalues, $v_{i}^{ \pm}$will denote the right eigenvectors $\left(1, \mu_{i}^{ \pm},\left(\mu_{i}^{ \pm}\right)^{2}\right)^{T}$ of $A^{ \pm}$, and $w_{i}^{ \pm}$the left eigenvectors such that $v_{i}^{ \pm} w_{i}^{ \pm}=1$.

The next lemma states the sign of $\operatorname{Re}\left(\mu_{i}^{+}\right)$and $\operatorname{Re}\left(\mu_{i}^{-}\right)$for $i=1,2,3$ for $\lambda$ in each of the connected components of $\mathbb{C} \backslash S^{+}$and $\mathbb{C} \backslash S^{-}$.

Lemma 2.2. For each $\tau>0$ fixed, the signs of the real part of the eigenvalues of $A^{ \pm}$change with $\lambda$ as follows:

$$
\begin{aligned}
& \text { If } \lambda \in \Omega_{r}^{+}, \quad \text { then } \operatorname{Re}\left(\mu_{1}^{+}(\lambda)\right)>0>\operatorname{Re}\left(\mu_{2}^{+}(\lambda)\right), \operatorname{Re}\left(\mu_{3}^{+}(\lambda)\right), \\
& \text { if } \lambda \in \Omega_{c}^{+}, \quad \text { then } 0>\operatorname{Re}\left(\mu_{1}^{+}(\lambda)\right) \geq \operatorname{Re}\left(\mu_{2}^{+}(\lambda)\right), \operatorname{Re}\left(\mu_{3}^{+}(\lambda)\right), \\
& \text { if } \lambda \in \Omega_{l}^{+}, \quad \text { then } \operatorname{Re}\left(\mu_{1}^{+}(\lambda)\right), \operatorname{Re}\left(\mu_{2}^{+}(\lambda)\right)>0>\operatorname{Re}\left(\mu_{3}^{+}(\lambda)\right) .
\end{aligned}
$$

Finally,

$$
\begin{aligned}
& \text { if } \lambda \in \Omega_{r}^{-}, \quad \text { then } \operatorname{Re}\left(\mu_{1}^{-}(\lambda)\right)>0>\operatorname{Re}\left(\mu_{2}^{-}(\lambda)\right), \operatorname{Re}\left(\mu_{3}^{-}(\lambda)\right), \\
& \text { if } \lambda \in \Omega_{l}^{-}, \quad \text { then } \operatorname{Re}\left(\mu_{1}^{-}(\lambda)\right), \operatorname{Re}\left(\mu_{2}^{-}(\lambda)\right)>0>\operatorname{Re}\left(\mu_{3}^{-}(\lambda)\right) .
\end{aligned}
$$

Proof. The result holds by application of the Routh-Hurwitz criterion, see for instance [4]. The method allows to count the number of roots with positive real part of a polynomial with real coefficients. Since in each simply connected component of $\mathbb{C} \backslash S^{ \pm}$the number of roots with positive (resp. negative) real part does not change and they intersect the real axis, we can apply this criterion for $\lambda$ real. This is done in detail in [8]. The sets $\Omega_{r, l, c}^{ \pm}$are depicted in Figure 1, as well as the sign of the real part of the roots of the polynomials $P_{\lambda}^{ \pm}$.

In order to locate the essential spectrum we observe that $(L-\lambda I)^{-1}$ is well defined in regions of the $\lambda$-plane that allow consistent splitting of roots, i.e regions where $P_{\lambda}^{-}$and $P_{\lambda}^{+}$have the same number of roots with positive (resp. negative) real part. This is because a Green's function for the operator $L-\lambda I$ can only be constructed by matching linearly independent solutions of (2.4) that decay to zero as $\eta \rightarrow+\infty$ to linearly independent solutions that decay to 0 as $\eta \rightarrow-\infty$. If for some $\lambda$ these solutions are linearly dependent, $\lambda$ is an eigenvalue. Thus eigenvalues must be isolated in regions of consistent splitting, whereas the rest of points are in the resolvent set. See e.g. $[\mathbf{2 6}],[\mathbf{1 2}],[\mathbf{1}],[\mathbf{1 5}]$.

In our case $\Omega_{r}^{-} \cap \Omega_{r}^{+}$and $\Omega_{l}^{-} \cap \Omega_{l}^{+}$are the regions of consistent splitting. The former contains $\mathbb{R}^{+} \backslash\{0\}$ and we denote it by

$$
\Omega:=\Omega_{r}^{-} \cap \Omega_{r}^{+} \text {. }
$$

We have the following

Proposition 2.3. The essential spectrum of $L$ is the set

$$
\mathbb{C} \backslash\left(\Omega \cup\left(\Omega_{l}^{-} \cap \Omega_{l}^{+}\right)\right)=\overline{\left(\Omega_{l}^{-} \cap \Omega_{r}^{+}\right) \cup\left(\Omega_{l}^{-} \cap \Omega_{c}^{+}\right)} .
$$

In principle, the real parts of $\mu_{2}^{ \pm}$and $\mu_{3}^{ \pm}$for $\lambda \in \Omega_{r}^{ \pm}$can coincide, this is an impediment to choosing solutions of (2.6) analytic in $\lambda \in \Omega_{r}^{ \pm}$that decay or grow exponentially with rates $\operatorname{Re}\left(\mu_{2}^{ \pm}\right)$or $\operatorname{Re}\left(\mu_{3}^{ \pm}\right)$. Next we show the presence of branch points for the eigenvalues of $A^{+}$with negative real part. 
LEMMA 2.4 ( $\mu^{+}$branch points). For $\tau>1$ there exists at least $a \lambda=\lambda_{b}(\tau) \in \Omega$ such that

$$
\mu_{2}^{+}\left(\lambda_{b}(\tau)\right)=\mu_{3}^{+}\left(\lambda_{b}(\tau)\right)
$$

PROOF. Real branch points are found by finding the critical points of

$$
\lambda(\mu)=\mu \frac{\tau \mu^{2}+\mu+1}{1-\tau \mu^{2}}
$$

that results from setting $P_{\lambda}^{+}=0$. For $\tau>1 / 4, \lambda(\mu)$ behaves like $\lambda(\mu) \rightarrow-\infty$ as $\mu \rightarrow+\infty$ and $\lambda(\mu) \rightarrow+\infty$ as $\mu \rightarrow-\infty$. It has a unique zero at $\mu=0$, two vertical asymptotes, at $\mu=-1 / \sqrt{\tau}$ and at $\mu=+1 / \sqrt{\tau}$, and an oblique one given by $\lambda=-\mu-1 / \tau$. The graph of $\lambda(\mu)$ is below $\lambda=-\mu-1 / \tau$ for $\mu>1 / \sqrt{\tau}$, and above it for $\mu<-1 / \sqrt{\tau}$, crossing it for $\mu \in(-1 / \sqrt{\tau,+1 / \sqrt{\tau}})$. Hence there are two real critical points. One, say $\mu_{r}$, with $\mu_{r}>1 / \sqrt{\tau}$ and $\lambda\left(\mu_{r}\right)<-\mu_{r}-1 / \tau<0$, the other, $\mu_{l}$, with $\mu_{l}<-1 / \sqrt{\tau}$ and $\lambda\left(\mu_{r}\right)>-\mu_{l}-1 / \tau$. The latter is clearly positive when $\tau>1$.

The following holds, see e.g. [4], [10], [17], see also Theorem A.4 and the following remarks in Appendix A.1:

Proposition 2.5. (i) For every $\lambda \in \Omega$ there exists a locally analytic in $\lambda$ base $\left(Y_{1}^{-}, Y_{2}^{-}, Y_{3}^{-}\right)$of fundamental solutions of (2.6) such that as $\eta \rightarrow-\infty, Y_{1}^{-}$and $\left(Y_{2}^{-}, Y_{3}^{-}\right)$span respectively the unstable manifold and stable manifolds of $d Y / d \eta=$ $A^{-} Y$. Similarly, there is a locally analytic in $\lambda$ base $\left(Y_{1}^{+}, Y_{2}^{+}, Y_{3}^{+}\right)$such that as $\eta \rightarrow+\infty, Y_{1}^{+}$and $\left(Y_{2}^{+}, Y_{3}^{+}\right)$span, respectively, the stable manifold and the unstable manifold of $d Y / d \eta=A^{+} Y$. The analogous statement is true for the adjoint system $d Z / d \eta=-Z A(\eta, \lambda)$.

(ii) The solutions spanning the one-dimensional manifolds (i.e. $Y_{1}^{-}, Y_{1}^{+}, Z_{1}^{+}$ and $Z_{1}^{-}$) are (globally) analytic in $\lambda \in \Omega$, and in particular

$$
\begin{array}{cc}
Y_{1}^{-}=e^{\mu_{1}^{-}(\lambda) \eta}\left(v_{1}^{-}+O\left(e^{-\alpha^{-}|\eta|}\right)\right) & \text { for } \eta \leq 0, \\
Z_{1}^{+}=e^{-\mu_{1}^{+}(\lambda) \eta}\left(w_{1}^{+}+O\left(e^{-\alpha^{+}|\eta|}\right)\right) & \text { for } \eta \geq 0 .
\end{array}
$$

The symbol $O$ here indicates, in each case, a vector function that is bounded in the above $\eta$-domain and that decays exponentially being $O\left(e^{-\alpha_{ \pm}|\eta|}\right)$ as $\eta \rightarrow \pm \infty$.

The presence of a branch point in $\Omega$ (Lemma 2.4), does not allow us to work globally in $\lambda$ with individual solutions $Y_{i}^{ \pm}$except for $Y_{1}^{ \pm}$. Instead the Green's function in Section 4 is constructed with exponential dichotomies to avoid this problem. Roughly speaking the dichotomies are the flows restricted to the solutions that approach the unstable or stable manifolds as $\eta \rightarrow \pm \infty$ of the corresponding limiting systems $d Y / d \eta=A^{ \pm} Y$. For the definition of exponential dichotomies and a discussion on the relation between them and bounded solutions we refer to Appendix A.1. We then have the following translation of Proposition 2.5 into the framework of exponential dichotomies that follows from Theorem A.5.

Proposition 2.6. For fixed $\lambda \in \Omega$, (2.6) has exponential dichotomies on $\mathbb{R}^{+}$ and on $\mathbb{R}^{-}$with exponential rates of decay bounded on $\lambda$. The dichotomies are analytic in $\lambda$ as long as $A^{ \pm}(\lambda)$ are hyperbolic, thus in particular in $\Omega$. 
In order to construct the exponential dichotomies uniquely, we define the projection $\mathcal{P}_{-}$such that if $\Phi(\eta, \xi)$ denotes the flow associated to $(2.6)$ with $\Phi(\xi, \xi)=I$, then for an initial condition $Y_{0} \in \mathbb{C}^{3}$ at $\eta=\xi$

$$
\mathcal{P}_{-}(\xi) Y_{0}=Y_{1}^{-}(\xi) \text {. }
$$

In other words $\mathcal{P}_{-}$projects any $Y \in \mathbb{C}^{3}$ into initial conditions for (2.6) that lead to a solution found in Proposition 2.5 that decays exponentially to 0 as $\xi \rightarrow-\infty$. We denote the flows of this dichotomy (see discussion before (A.13)) by

$$
\Phi_{-}^{u}(\eta, \xi)=\Phi(\eta, \xi) \mathcal{P}_{-}(\xi), \quad \Phi_{-}^{s}(\eta, \xi):=\Phi(\eta, \xi)\left(I-\mathcal{P}_{-}(\xi)\right)
$$

Analogously, the exponential dichotomy defined on $[0,+\infty)$ is fixed by introducing the projection $\mathcal{P}_{+}$such that for an initial condition $Y_{0} \in \mathbb{C}^{3}$ at $\eta=\xi$

$$
\mathcal{P}_{+}(\xi) Y_{0}=Y_{1}^{+}(\xi)
$$

and we denote the flows by

$$
\Phi_{+}^{u}(\eta, \xi):=\Phi(\eta, \xi) \mathcal{P}_{+}(\xi), \quad \Phi_{+}^{s}(\eta, \xi):=\Phi(\eta, \xi)\left(I-\mathcal{P}_{+}(\xi)\right) .
$$

We stress that $\mathcal{P}_{-}$and $\mathcal{P}_{+}$are well defined on $\mathbb{R}$, and equally are the partial flows $\Phi_{ \pm}^{u, s}$, what is not guaranteed in the whole of $\mathbb{R}$ is that they give exponential dichotomies. We have the following relation between the spectrum of the operator $L$ and the existence of exponential dichotomies of (2.6). The proof can be found in [18] and [19], see [25] for a discussion in the present context.

LEMMA 2.7. $\lambda \in \mathbb{C}$ is in the resolvent of $L$ if and only if (2.6) has an exponential dichotomy on $\mathbb{R}$. $\lambda$ is in the point spectrum of $L$ if (2.6) has exponential dichotomies on $\mathbb{R}^{+}$and on $\mathbb{R}^{-}$with the same Morse index, but neither can be extended to an exponential dichotomy on $\mathbb{R}$ (i.e. (2.6) must have bounded solutions on $\mathbb{R}$ ). $\lambda \in \mathbb{C}$ is in the essential spectrum if (2.6) either does not have a exponential dichotomy on $\mathbb{R}^{+}$and/or on $\mathbb{R}^{-}$, or it does but they have different Morse index.

We now define the Evans function. We follow the idea in [21]. We take the Evans function $D(\lambda)$ to be the transmission coefficient such that for all $\lambda \in \Omega$

$$
Y_{1}^{-}(\eta, \lambda) \sim D(\lambda) e^{\mu_{1}^{+}(\lambda) \eta} v_{1}^{+} \quad \text { as } \eta \rightarrow+\infty
$$

Clearly by Lemma 2.2 , if $D(\lambda)=0$, then $Y_{1}^{-}$decays to 0 as $|\eta| \rightarrow+\infty$, therefore $\lambda$ is an eigenvalue and $Y_{1}^{-}$its corresponding eigenfunction. Conversely, if $Y$ is an eigenfunction for some $\lambda \in \Omega$, then $Y$ must be a multiple of $Y_{1}^{-}$. This is because other independent solutions of (2.4) do not decay to 0 as $\eta \rightarrow-\infty$. $D(\lambda)$ can be expressed as the scalar product

$$
D(\lambda):=Z_{1}^{+} Y_{1}^{-}
$$

see [21]. For alternative, but equivalent definitions see for instance [1] and [15].

The following properties are satisfied by the Evans function.

LEMMA 2.8. (i) $D(\lambda)$ does not depend on $\eta$. (ii) $D(\lambda)$ is analytic in the domain $\Omega:=\Omega_{r}^{+} \cap \Omega_{r}^{-}$. (iii) Also $\overline{D(\lambda)}=D(\bar{\lambda})$.

The first statement is immediate from the formulation (2.18). Analyticity holds by standard arguments for ODE's and (2.18). The last statement holds by using the symmetry $A(\eta, \bar{\lambda})=\overline{A(\eta, \lambda)}$. 
The function $D(\lambda)$ can be extended through the essential spectrum to a neighbourhood of $\lambda=0$, in fact to a neighbourhood of $S^{-}$, as it is shown in the next lemma.

Lemma 2.9. There exists an open set $\Omega^{*}$ in $\mathbb{C}$ that contains the sets $S^{-}$and $\Omega$, such that for all $\tau>0, D(\lambda)$ is well-defined and analytic in $\Omega^{*}$.

Proof. Observe that $D(\lambda)$ is well-defined for all $\lambda$ such that

$$
\begin{gathered}
\operatorname{Re}\left(\mu_{1}^{+}(\lambda)\right)>\operatorname{Re}\left(\mu_{2}^{+}(\lambda)\right), \operatorname{Re}\left(\mu_{3}^{+}(\lambda)\right), \\
\operatorname{Re}\left(\mu_{1}^{-}(\lambda)\right)>\operatorname{Re}\left(\mu_{2}^{-}(\lambda)\right), \operatorname{Re}\left(\mu_{3}^{-}(\lambda)\right) .
\end{gathered}
$$

These conditions are satisfied for $\lambda \in S^{-}$. Indeed, if $\lambda \in S^{-}$, then $\lambda=\frac{-s^{2}}{1+\tau s^{2}}-i s$ for $s \in \mathbb{R}$, and the roots of $P_{\lambda}^{-}$are

$$
\mu_{1}^{-}(\lambda)=\frac{-1+\Delta^{\frac{1}{2}}}{2 \tau}, \mu_{2}^{-}(\lambda)=s i, \mu_{3}^{-}(\lambda)=\frac{-1-\Delta^{\frac{1}{2}}}{2 \tau},
$$

where $\Delta:=1+4 \tau\left(\tau s^{2}+1+\tau^{2} s^{2}\right)-4 i s \tau\left(1+s^{2} \tau\right)$, with $\operatorname{Re}\left(\Delta^{\frac{1}{2}}\right)>1$, this implies (2.20). Also if $\lambda \in S^{-} \backslash\{0\}$, then $\lambda \in \Omega_{r}^{+}$, and (2.19) holds by Lemma 2.2. Finally if $\lambda=0$,

$$
\mu_{1}^{+}(0)=0, \mu_{2}^{+}(0)=\frac{-1+(1-4 \tau)^{\frac{1}{2}}}{2 \tau}, \mu_{3}^{+}(0)=\frac{-1-(1-4 \tau)^{\frac{1}{2}}}{2 \tau},
$$

hence (2.19) also holds. Since $\mu_{1}^{ \pm}$are analytic in $\lambda \in \bar{\Omega}$, there exists a neighbourhood $U$ of $S^{-}$, in which (2.19) and (2.20) hold, and hence $D(\lambda)$ is well-defined.

As a consequence, for all $\tau>1 / 4$ and if $\Omega^{*}$ is an open set as in Lemma 2.9, there exist a $\delta_{\tau}>0$ small enough such that

$$
\Omega^{* *}:=\left\{\lambda \in \mathbb{C}: \operatorname{Re}(\lambda)>-\delta_{\tau}\right\} \subset \Omega^{*}
$$

and $D(\lambda)$ is well-defined and analytic in $\Omega^{* *}$.

Then $D(0)$ is well-defined and clearly $D(0)=0$. The following lemma gives the value of $D^{\prime}(0)$, a result that will be used in Section 6 .

Lemma 2.10. For all $\tau>0, \lambda=0$ is a simple zero of $D(\lambda)$, with

$$
D^{\prime}(0)=\frac{2 \tau}{(-1+\sqrt{1+4 \tau})}>0
$$

The proof is analogous to that in $[\mathbf{2 1}]$ and can be found in $[\mathbf{8}]$.

\section{Large $|\lambda|$}

In this section we analyse the behaviour of $D(\lambda)$ and of the solutions to (2.6) as $|\lambda| \rightarrow+\infty$. We establish the existence of dichotomies in this regime and provide the key estimates on them. The estimates will be needed in Section 4 and that on $D$ will be used in Section 6. Throughout this section the value of $\tau$ will be fixed with $\tau>1 / 4$ unless otherwise specified. We also assume that $\lambda \in \Omega^{* *}$ as defined in $(2.21)$.

We work in the framework of exponential dichotomies. The arguments underlying the behaviour of the solutions to (2.6) are similar to that in [17] (in particular A.3.2.3) and are inspired in the theory of (perturbed) linear ODE's (see [4]). The key observation is that for $\lambda \in \Omega^{* *}$ with $|\lambda|$ large enough there are three distinct eigenvalues of the matrix $A$ with the ordering or real parts as for $A^{+}$and $A^{-}$in 
the domains $\Omega_{r}^{+}$and $\Omega_{r}^{-}$respectively. Diagonalising $A$ allows to neatly conclude that there is an exponential dichotomy on $\mathbb{R}$. Then estimates on the partial flows for large $|\lambda|$ are derived using Theorem A.3 of the Appendix.

Let us introduce some notation. Let the characteristic polynomial of $A$ be denoted by

$$
P_{\lambda}(\mu, \eta):=\tau \mu^{3}+(1+\tau \lambda) \mu^{2}+(2 \phi(\eta)-1) \mu+\left(2 \phi^{\prime}(\eta)-\lambda\right),
$$

and its derivative with respect to $\mu$ by

$$
P_{\lambda}^{\prime}(\mu, \eta):=3 \tau \mu^{2}+2(1+\tau \lambda) \mu+(2 \phi(\eta)-1) .
$$

Thus, denoting by $B_{M}(0)$ a ball in $\mathbb{C}$ of radius $M$ and centre $\lambda=0$, we have

LEMma 3.1. There exist $M>0$ large enough such that if $\lambda \in \Omega^{* *} \backslash B_{M}(0)$ then for all $\eta \in \mathbb{R}$ the eigenvalues of $A \mu_{1}(\eta, \lambda), \mu_{2}(\eta, \lambda)$ and $\mu_{3}(\eta, \lambda)$ satisfy

$$
\operatorname{Re}\left(\mu_{1}(\eta, \lambda)\right) \geq \kappa_{u}>0>\kappa_{s} \geq \operatorname{Re}\left(\mu_{2}(\eta, \lambda)\right), \operatorname{Re}\left(\mu_{3}(\eta, \lambda)\right),
$$

for some $\kappa_{u}$ and $\kappa_{s}$ (depending on $\tau$ and $\left.M\right)^{3}$. Also $\mu_{3} \neq \mu_{2}$ and they behave asymptotically like

$$
\begin{aligned}
& \mu_{1}(\eta, \lambda)=+\frac{1}{\sqrt{\tau}}-\left(\frac{1}{\tau}+\frac{2}{\sqrt{\tau}} \phi(\eta)+2 \phi^{\prime}(\eta)\right) \frac{1}{2 \sqrt{\tau} \lambda}+O\left(|\lambda|^{-2}\right) \\
& \mu_{2}(\eta, \lambda)=-\frac{1}{\sqrt{\tau}}+\left(\frac{1}{\tau}-\frac{2}{\sqrt{\tau}} \phi(\eta)+2 \phi^{\prime}(\eta)\right) \frac{1}{2 \sqrt{\tau} \lambda}+O\left(|\lambda|^{-2}\right) \\
& \mu_{3}(\eta, \lambda)=-\lambda-\frac{1}{\tau}+\frac{2}{\tau} \phi(\eta) \frac{1}{\lambda}+O\left(|\lambda|^{-2}\right),
\end{aligned}
$$

as $|\lambda| \rightarrow \infty$ for $\lambda \in \Omega$ and uniformly in $\eta \in \mathbb{R}$.

Proof. We let $\delta:=|\lambda|^{-1}$, and $\theta:=\arg \lambda$. For $\delta=0$ the polynomial $P_{\lambda}$ reduces to $\tau \mu^{2}-1=0$ which has the simple roots $\mu=+1 / \sqrt{\tau}$ and $\mu=-1 / \sqrt{\tau}$. Using the implicit function theorem in a small neighbourhood of $\delta=0$, we can introduce an expansion $\mu=\mu^{0}+\mu^{1} \delta+O\left(\delta^{2}\right)$ as $\delta \rightarrow 0$, to get (3.3) and (3.4). The argument is applied first point-wise in $\eta$. The uniformity follows from the regularity of $\phi$ and $\phi^{\prime}$ and the fact that they are uniformly bounded in $\eta \in \mathbb{R}$.

In order to capture the third eigenvalue we scale the polynomial $P$ by setting $\tilde{\mu}=\delta^{\alpha} \mu$, with $\alpha>0$, then,

$\delta^{3 \alpha} P_{\lambda}\left(\frac{\tilde{\mu}}{\delta^{\alpha}}, \eta\right)=\tau \tilde{\mu}^{3}+\left(1+\tau \frac{e^{i \theta}}{\delta}\right) \tilde{\mu}^{2} \delta^{\alpha}+(2 \phi(\eta)-1) \tilde{\mu} \delta^{2 \alpha}-e^{i \theta} \delta^{3 \alpha-1}+2 \phi^{\prime}(\eta) \delta^{3 \alpha}$

For $\tau$ fixed, the only uniform balance comes when the third order term and the second order term dominate, thus taking $\alpha=1$ the reasoning above gives that there is a root with behaviour

$$
\tilde{\mu}=-e^{i \theta}-\frac{1}{\tau} \delta+\frac{2}{\tau} \phi(\eta) e^{-i \theta} \delta^{2}+O\left(\delta^{3}\right) .
$$

For $M>0$ as in the previous lemma and with $\lambda \in \Omega^{* *} \backslash B_{M}(0)$ we set the notation

$$
v_{i}(\eta, \lambda):=\left(1, \mu_{i}(\eta, \lambda),\left(\mu_{i}(\eta, \lambda)\right)^{2}\right)^{T} \quad i=1,2,3
$$

\footnotetext{
${ }^{3}$ In fact $\kappa_{u}, \kappa_{s}$ tend to 0 as $\tau \rightarrow \infty$
} 
(that are the right eigenvectors of $A(\eta, \lambda)$ ), and for each $i=1,2,3$ with $j$ and $k$ in $\{1,2,3\}, j, k \neq i$ and $j \neq k$

$$
w_{i}(\eta, \lambda):=\frac{\tau}{P_{\lambda}^{\prime}\left(\mu_{i}(\eta, \lambda), \eta\right)}\left(\mu_{k}(\eta, \lambda) \mu_{j}(\eta, \lambda),-\mu_{j}(\eta, \lambda)-\mu_{k}(\eta, \lambda), 1\right),
$$

(the left eigenvectors). Here $w_{i}$ and $v_{i}$ are normalised so that the matrices

$$
V:=\left(v_{1}, v_{2}, v_{3}\right) \quad \text { and } \quad W:=\left(w_{1}, w_{2}, w_{3}\right)^{T} .
$$

satisfy $W V=I$.

For the argument that follows we need bounds on the entries of $W$ and $V$ and on the matrix $W d V / d \eta$. As a final preparation we compute

$$
W \frac{d V}{d \eta}=\left(\begin{array}{lll}
r_{11} & r_{12} & r_{13} \\
r_{21} & r_{22} & r_{23} \\
r_{31} & r_{32} & r_{33}
\end{array}\right)
$$

with

$$
r_{i j}:=\frac{1}{P_{\lambda}^{\prime}\left(\mu_{i}\right)}\left(\mu_{i}+\frac{1}{\tau}+\lambda+2 \mu_{j}\right) \frac{d \mu_{i}}{d \eta} \text { for all } i, j=1,2,3 .
$$

And the estimates on $V, W$ and $W d V / d \eta$ are a consequence of Lemma 3.1, namely

Corollary 3.2. For $\lambda \in \Omega^{* *}$ with $|\lambda|>M$, for $M$ large enough, the matrix functions $V$ and $W$ are uniformly bounded in $\xi \in \mathbb{R}$ but not in $\lambda$, with expansions as $|\lambda| \rightarrow \infty$

$$
\begin{aligned}
& v_{1}(\eta, \lambda)=\left(1, \frac{1}{\sqrt{\tau}}+O\left(|\lambda|^{-1}\right), \frac{1}{\tau}+O\left(|\lambda|^{-1}\right)\right) \\
& v_{2}(\eta, \lambda)=\left(1,-\frac{1}{\sqrt{\tau}}+O\left(|\lambda|^{-1}\right), \frac{1}{\tau}+O\left(|\lambda|^{-1}\right)\right) \\
& v_{3}(\eta, \lambda)=\left(1,-\lambda-\frac{1}{\tau}+O\left(|\lambda|^{-1}\right), \lambda^{2}+2 \tau \lambda-4 \phi(\eta)+O\left(|\lambda|^{-1}\right)\right)
\end{aligned}
$$

and

$$
\begin{aligned}
& w_{1}(\eta, \lambda)=\left(\frac{1}{2}+O\left(|\lambda|^{2}\right), \frac{1}{2} \sqrt{\tau}+O\left(|\lambda|^{-1}\right), \frac{1}{2} \sqrt{\tau} \frac{1}{\lambda}+O\left(|\lambda|^{-2}\right)\right)^{T}, \\
& w_{2}(\eta, \lambda)=\left(\frac{1}{2}+O\left(|\lambda|^{2}\right),-\frac{1}{2} \sqrt{\tau}+O\left(|\lambda|^{-1}\right),-\frac{1}{2} \sqrt{\tau} \frac{1}{\lambda}+O\left(|\lambda|^{-2}\right)\right)^{T}, \\
& w_{3}(\eta, \lambda)=\left(-\frac{1}{\tau} \frac{1}{\lambda^{2}}+O\left(|\lambda|^{3}\right),-\frac{2 \phi(\eta)}{\tau} \frac{1}{\lambda^{3}}+O\left(|\lambda|^{-4}\right), \frac{1}{\lambda^{2}}+O\left(|\lambda|^{-3}\right)\right)^{T} .
\end{aligned}
$$

Moreover, the elements of the matrix function $W V^{\prime}$ satisfy

$$
\left|r_{i j}(\eta, \lambda)\right| \leq C(\lambda) e^{-\alpha|\eta|} \quad \text { for all } \quad \eta \in \mathbb{R}, \quad \alpha=\min \left\{\alpha^{+}, \alpha^{-}\right\}
$$

for a constant $C$ such that as $|\lambda| \rightarrow \infty$, the elements of $W V^{\prime}$ have

$$
\begin{array}{lll}
r_{11}(\eta, \lambda)=O\left(|\lambda|^{-1}\right), & r_{12}(\eta, \lambda)=O\left(|\lambda|^{-1}\right), & r_{13}(\eta, \lambda)=O\left(|\lambda|^{-1}\right) \\
r_{21}(\eta, \lambda)=O\left(|\lambda|^{-1}\right), & r_{22}(\eta, \lambda)=O\left(|\lambda|^{-1}\right), & r_{23}(\eta, \lambda)=O\left(|\lambda|^{-1}\right) \\
r_{31}(\eta, \lambda)=O\left(|\lambda|^{-3}\right), & r_{32}(\eta, \lambda)=O\left(|\lambda|^{-3}\right), & r_{33}(\eta, \lambda)=O\left(|\lambda|^{-2}\right)
\end{array} .
$$


The proof, although arduous, follows easily and we shall not write it down. The important thing to notice of this result is that all the elements of $W d V / d \eta$ are small as $|\lambda| \rightarrow \infty$.

The crucial estimates are the following.

ThEOREM 3.3. (i) Let $\tau>0$ and $\lambda \in \Omega$, then

$$
D(\lambda) \rightarrow 1 \quad \text { as } \quad|\lambda| \rightarrow \infty
$$

And there exists a $M>0$ large enough such that $\Omega^{* *} \backslash B_{M}(0)$ contains no eigenvalues of $L$.

(ii) For $\lambda \in \Omega^{* *} \backslash B_{M}(0)$ with $M>0$ large enough there is an exponential dichotomy of (2.6) on $\mathbb{R}$, with partial flows, denoted by $\Phi^{s}$ and $\Phi^{u}$, such that there exist $a \delta>0$ small enough with $\kappa_{u}-\delta>0$ and $\kappa_{s}+\delta<0$ and $a K(\lambda)>0$ (unbounded as $|\lambda| \rightarrow \infty$ ) such that

$$
\left|\Phi^{u}(\eta, \xi)\right| \leq K(\lambda) e^{\left(\kappa_{u}-\delta\right)(\eta-\xi)} \quad \eta \leq \xi,\left|\Phi^{s}(\eta, \xi)\right| \leq K(\lambda) e^{\left(\kappa_{s}+\delta\right)(\eta-\xi)} \quad \eta \geq \xi .
$$

Moreover, there exists a constant $K>0$ such that the following elements of the operator matrices $\Phi^{s}$ and $\Phi^{u}$ satisfy, for all $\eta \leq \xi$ for the elements of $\Phi^{u}$ and for all $\eta \geq \xi$ for the ones of $\Phi^{s}$,

$$
\begin{gathered}
\left|\Phi_{12}^{u}(\eta, \xi)\right| \leq K e^{\left(\kappa_{u}-\delta\right)(\eta-\xi)}, \quad\left|\Phi_{12}^{s}(\eta, \xi)\right| \leq K e^{\left(\kappa_{s}+\delta\right)(\eta-\xi)} . \\
\left|\Phi_{13}^{u}(\eta, \xi)\right| \leq \frac{K}{|\lambda|} e^{\left(\kappa_{u}-\delta\right)(\eta-\xi)}, \quad\left|\Phi_{13}^{s}(\eta, \xi)\right| \leq \frac{K}{|\lambda|} e^{\left(\kappa_{s}+\delta\right)(\eta-\xi)}, \\
\left|\Phi_{22}^{u}(\eta, \xi)\right| \leq K e^{\left(\kappa_{u}-\delta\right)(\eta-\xi)}, \quad\left|\Phi_{22}^{s}(\eta, \xi)\right| \leq K e^{\left(\kappa_{s}+\delta\right)(\eta-\xi)} . \\
\left|\Phi_{23}^{u}(\eta, \xi)\right| \leq \frac{K}{|\lambda|} e^{\left(\kappa_{u}-\delta\right)(\eta-\xi)}, \quad\left|\Phi_{23}^{s}(\eta, \xi)\right| \leq \frac{K}{|\lambda|} e^{\left(\kappa_{s}+\delta\right)(\eta-\xi)} . \\
\left|\Phi_{33}^{u}(\eta, \xi)\right| \leq K e^{\left(\kappa_{u}-\delta\right)(\eta-\xi)}, \quad\left|\Phi_{33}^{s}(\eta, \xi)\right| \leq K e^{\left(\kappa_{s}+\delta\right)(\eta-\xi)} .
\end{gathered}
$$

Moreover, although $\Phi_{32}^{s, u}$ are not uniformly bounded in $\lambda$, the following combination is

$$
\begin{aligned}
& \left|\Phi_{32}^{u}(\eta, \xi)-\left(\lambda+\frac{1}{\tau}\right) \Phi_{33}^{u}(\eta, \xi)\right| \leq e^{\left(\kappa_{u}-\delta\right)(\eta-\xi)} K \quad \eta \leq \xi \\
& \left|\Phi_{32}^{s}(\eta, \xi)-\left(\lambda+\frac{1}{\tau}\right) \Phi_{33}^{s}(\eta, \xi)\right| \leq e^{\left(\kappa_{s}+\delta\right)(\eta-\xi)} K \quad \eta \geq \xi
\end{aligned}
$$

Proof. (i) That $D(\lambda) \rightarrow 1$ was proved in [8] following the ideas in [21]. We shall not give the proof here.

(ii) We write the equation for $\mathcal{Y}:=W Y$, where $Y$ satisfies (2.6). For that we compute

$$
\frac{d(W Y)}{d \eta}=W \frac{d Y}{d \eta}+\frac{d W}{d \eta} Y=W A Y+\frac{d W}{d \eta} Y=W A V(W Y)+\frac{d W}{d \eta} V(W Y)
$$

and observing that $d(W V) / d \eta=0$ and that $W A V=\operatorname{diag}\left(\mu_{1}, \mu_{2}, \mu_{3}\right)$ we get that $\mathcal{Y}$ satisfies

$$
\frac{d \mathcal{Y}}{d \eta}=\operatorname{diag}\left(\mu_{1}, \mu_{2}, \mu_{3}\right) \mathcal{Y}-W \frac{d V}{d \eta} \mathcal{Y}
$$


Thus, by Corollary 3.2, (3.10) can be seen as a $O\left(|\lambda|^{-1}\right)$-perturbation of the system

$$
\frac{d \mathcal{Y}}{d \eta}=\operatorname{diag}\left(\mu_{1}, \mu_{2}, \mu_{3}\right) \mathcal{Y}
$$

For $\lambda \in \Omega^{* *} \backslash B_{M}(0)$ the roots $\mu_{i}$ are simple for all $\eta \in \mathbb{R}$ and thus the spectral projection associated to $\mu_{1}$ is clearly

$$
\mathcal{P}_{u n}(\xi) \equiv\left(\begin{array}{ccc}
1 & 0 & 0 \\
0 & 0 & 0 \\
0 & 0 & 0
\end{array}\right)
$$

We denote the complementary projection of $\mathcal{P}_{\text {un }}$ by $\mathcal{Q}_{\text {un }}=I-\mathcal{P}_{\text {un }}$, and observe that the flow associated to (3.11), which is simply

$$
\Phi_{u n}(\eta, \xi)=\exp \left(\int_{\xi}^{\eta}\left(\begin{array}{ccc}
\mu_{1}(s, \lambda) & 0 & 0 \\
0 & \mu_{2}(s, \lambda) & 0 \\
0 & 0 & \mu_{3}(s, \lambda)
\end{array}\right) d s\right)
$$

defines an exponential dichotomy for (3.11), namely

$$
\begin{gathered}
\left|\Phi_{u n}(\eta, \xi) \mathcal{P}_{u n}(\xi)\right| \leq K e^{\kappa_{u}(\eta-\xi)} \quad \eta \leq \xi \\
\left|\Phi_{u n}(\eta, \xi) \mathcal{Q}_{u n}(\xi)\right| \leq K e^{\kappa_{s}(\eta-\xi)} \quad \eta \geq \xi
\end{gathered}
$$

with $\kappa_{u}$ and $\kappa_{s}$ as in Lemma 3.1. Provided that $M$ is large enough, Theorem A.3 of the Appendix A.1 implies that (3.10) has exponential dichotomies $\Phi_{*}^{u}$ and $\Phi_{*}^{s}$ such that there exists a $\delta>0$, with $\delta=O\left(|\lambda|^{-1}\right)$ as $|\lambda| \rightarrow \infty$, and a constant $K_{*}$ with

$$
\begin{array}{ll}
\left|\Phi_{*}^{u}(\eta, \xi)\right| \leq K_{*} e^{\left(\kappa_{u}-\delta\right)(\eta-\xi)} & \eta \leq \xi \\
\left|\Phi_{*}^{s}(\eta, \xi)\right| \leq K_{*} e^{\left(\kappa_{s}+\delta\right)(\eta-\xi)} & \eta \geq \xi
\end{array}
$$

It is not difficult to prove using the representation of the partial flows (given in the Appendix) (A.9)-(A.10) on $\mathbb{R}$, that the dichotomies of (3.10) and of (3.11) are $|\lambda|^{-1}$ close. Namely, there exist positive constant $C$ depending only on $M$ such that

$$
\begin{aligned}
\left|\Phi_{*}^{u}(\eta, \xi)-\Phi_{u n}(\eta, \xi) \mathcal{P}_{u n}(\xi)\right| \leq \frac{C}{|\lambda|} e^{\left(\kappa_{u}-\delta\right)(\eta-\xi)} & \eta \leq \xi \\
\left|\Phi_{*}^{s}(\eta, \xi)-\Phi_{u n}(\eta, \xi) \mathcal{Q}_{u n}(\xi)\right| \leq \frac{C}{|\lambda|} e^{\left(\kappa_{s}+\delta\right)(\eta-\xi)} & \eta \geq \xi .
\end{aligned}
$$

Observe that $\Phi(\eta, \xi)=V(\eta)\left(\Phi_{*}^{s}(\eta, \xi)+\Phi_{*}^{u}(\eta, \xi)\right) W(\xi)$ gives the flow associated to $(2.6)$ with $\Phi(\eta, \eta) \equiv I$. In fact the partial flows

$$
\Phi^{s}(\eta, \xi):=V(\eta) \Phi_{*}^{s}(\eta, \xi) W(\xi), \quad \Phi^{u}(\eta, \xi):=V(\eta) \Phi_{*}^{u}(\eta, \xi) W(\xi)
$$

define an exponential dichotomy of (2.6). To see this we check that Definition A.2 of the Appendix is satisfied. It is straightforward to prove that the projection

$$
\mathcal{Q}(\eta)=\Phi^{s}(\eta, \xi)
$$

commutes with the flow. This proves (ii) of Definition A.2. The of proof (i) (that is the exponential decay estimates analogous to (3.12) and (3.13)) follows by realising that $V$ and $W$ are uniformly bounded with respect to $\eta$. 
We now get the estimates for the elements of the flows outlined in the statement of this theorem. We only write the estimate on $\Phi_{32}^{u}-(\lambda+1 / \tau) \Phi_{33}^{u}$ in some detail. First we recall that

$$
\Phi^{u}(\eta, \xi)-V(\eta) \Phi_{u n}(\eta, \xi) \mathcal{P}_{u n}(\xi) W(\xi)=V(\eta)\left(\Phi_{*}^{u}(\eta, \xi)-\Phi_{u n}(\eta, \xi) \mathcal{P}_{u n}(\xi)\right) W(\xi)
$$

and that, by (3.14), all elements of $\Phi_{*}^{u}$ have

$$
\left|\Phi_{*, i j}^{u}(\eta, \xi)-\left(\Phi_{u n}(\eta, \xi) \mathcal{P}_{u n}(\xi)\right)_{i j}\right|=\left|\Phi_{*, i j}^{u}(\eta, \xi)\right| \leq \frac{C}{|\lambda|} e^{\left(\kappa_{u}-\delta\right)(\eta-\xi)} \quad \eta \leq \xi
$$

except for

$$
\left|\Phi_{*, 11}^{u}(\eta, \xi)-\left(\Phi_{u n}(\eta, \xi) \mathcal{P}_{u n}(\xi)\right)_{i j}\right|=\left|\Phi_{*, 11}^{u}(\eta, \xi)-e^{\int_{\xi}^{\eta} \mu_{1}(s, \lambda) d s}\right| \leq \frac{C}{|\lambda|} e^{\left(\kappa_{u}-\delta\right)(\eta-\xi)}
$$

From (3.16) we compute $\Phi_{32}^{u}$ and $\Phi_{33}^{u}$, and so

$$
\begin{aligned}
\Phi_{32}^{u}-(\lambda+1 / \tau) \Phi_{33}^{u} & =\left(\sum_{i=1}^{3} \Phi_{*, i 1}^{u}(\eta, \xi) v_{i}^{3}(\eta, \lambda)\right)\left(w_{1}^{2}(\xi, \lambda)-(\lambda+1 / \tau) w_{1}^{3}(\xi, \lambda)\right) \\
& +\left(\sum_{i=1}^{3} \Phi_{*, i 2}^{u}(\eta, \xi) v_{i}^{3}(\eta, \lambda)\right)\left(w_{2}^{2}(\xi, \lambda)-(\lambda+1 / \tau) w_{2}^{3}(\xi, \lambda)\right) \\
& +\left(\sum_{i=1}^{3} \Phi_{*, i 3}^{u}(\eta, \xi) v_{i}^{3}(\eta, \lambda)\right)\left(w_{3}^{2}(\xi, \lambda)-(\lambda+1 / \tau) w_{3}^{3}(\xi, \lambda)\right),
\end{aligned}
$$

where $w_{i}^{j}$ is the $j$-th coordinate of $w_{i}$ and $v_{i}^{3}$ is the third coordinate of $v_{i}$ for $i=1,2,3$. The result follows by applying Corollary 3.2. The not so obvious estimates are for those terms involving $v_{3}^{3}$ since this is $O\left(|l|^{2}\right)\left(v_{1}^{3}\right.$ and $v_{2}^{3}$ are $\left.O(1)\right)$ as $|\lambda| \rightarrow+\infty$, but a simple computation shows that each of the factors $w_{i}^{2}(\xi, \lambda)-(\lambda+$ $\left.1 / \tau) w_{i}^{3}(\xi, \lambda)\right)=O\left(|\lambda|^{-1}\right)$ with $i=1,2,3$ which together with the above observation on the elements on $\Phi_{*}^{u}$ leads to the desired estimate.

\section{Analysis of the resolvent kernel}

Throughout we assume that for $\lambda \in \Omega^{* *} D(\lambda) \neq 0$ except for $\lambda=0$. We construct the Green's function associated to (2.5) in a standard way below. For the moment let it be denoted by $G_{\lambda}(\eta, \xi)$, then for any given $f \in L^{2}(\mathbb{R}), u \in H^{2}(\mathbb{R})$ given by

$$
u(\eta)=\int_{\mathbb{R}} G_{\lambda}(\eta, \xi) f(\xi) d \xi
$$

solves $\left(\tilde{L}-\lambda\left(I-\tau \frac{d^{2}}{d \eta^{2}}\right)\right) u=f$. But we have to solve the resolvent problem for $L$. Clearly,

$$
u(\eta)=\int_{\mathbb{R}} G_{\lambda}(\eta, \xi)\left(I-\tau \frac{d^{2}}{d \xi^{2}}\right) f(\xi) d \xi \in H^{2}(\mathbb{R}),
$$

gives the solution to $(L-\lambda) u=f$ for $f \in H^{2}(\mathbb{R})$.

To compute the Green's function associated to (2.5) we use the variation of constant formula for the inhomogeneous problem

$$
\frac{d}{d \eta} Y(\eta)=A(\eta, \lambda) Y(\eta)+(0,0, \delta(\eta-\xi))^{T}
$$


for any fixed $\xi$ in $\mathbb{R}$. With the usual notation for flows and matrices of fundamental solutions we obtain the solution

$$
\mathcal{G}_{\lambda}(\eta, \xi)=\left\{\begin{array}{rr}
\frac{1}{2} \Phi(\eta, \xi)\left((0,0,1)^{T}+2 K^{+}(\xi)\right), & \eta>\xi \\
-\frac{1}{2} \Phi(\eta, \xi)\left((0,0,1)^{T}-2 K^{-}(\xi)\right), & \eta<\xi
\end{array}\right.
$$

where the vectors $K^{+}$and $K^{-}$are determined by imposing the jump condition

$$
\mathcal{G}_{\lambda}\left(\xi^{+}, \xi\right)-\mathcal{G}_{\lambda}\left(\xi^{-}, \xi\right)=(0,0,-1)^{T}
$$

and the asymptotic behaviour

$$
\mathcal{G}_{\lambda}(\eta, \xi) \rightarrow(0,0,0)^{T} \quad \text { as } \quad|\eta| \rightarrow \infty \text { for all } \xi \in \mathbb{R} .
$$

The conditions (4.2) and (4.3) respectively imply that

$$
K^{+}(\xi)-K^{-}(\xi)=(0,0,-2)^{T},
$$

and, recalling the projections $(2.13)$ and $(2.15)$,

$(0,0,1 / 2)+K^{+}(\xi) \propto\left(I-\mathcal{P}_{+}(\xi)\right)(0,0,1)^{T} \quad$ and $\quad(0,0,1 / 2)-K^{+}(\xi) \propto \mathcal{P}_{-}(\xi)(0,0,1)^{T}$.

Hence

$$
\begin{aligned}
K^{+}(\xi) & =-\left(I-\mathcal{P}_{+}(\xi)\right)(0,0,1)^{T}-(0,0,1 / 2) \\
K^{-}(\xi) & =\mathcal{P}_{-}(\xi)(0,0,1)^{T}+(0,0,1 / 2)^{T} .
\end{aligned}
$$

One finally obtains

$$
\mathcal{G}_{\lambda}(\eta, \xi)=\left\{\begin{aligned}
-\Phi_{+}^{s}(\eta, \xi)(0,0,1)^{T}, & \eta>\xi \\
\Phi_{-}^{u}(\eta, \xi)(0,0,1)^{T}, & \eta<\xi
\end{aligned}\right.
$$

We also compute $\partial \mathcal{G}_{\lambda}(\eta, \xi) / \partial \xi$. We need to get estimates of these derivatives of $G_{\lambda}$ to provide the estimates on the $H^{2}$-norm of (4.1). It follows from $\partial \Phi(\eta, \xi) / \partial \xi=$ $-\Phi(\eta, \xi) A(\xi, \lambda)$ and from $A(\xi, \lambda)(0,0,1)^{T}=(0,1,-(\lambda+1 / \tau))^{T}$ that

$$
\frac{\partial}{\partial \xi} \mathcal{G}_{\lambda}(\eta, \xi)=\left\{\begin{aligned}
\Phi_{+}^{s}(\eta, \xi)(0,1,-(\lambda+1 / \tau))^{T}, & \eta>\xi \\
-\Phi_{-}^{u}(\eta, \xi)(0,1,-(\lambda+1 / \tau))^{T}, & \eta<\xi
\end{aligned}\right.
$$

with the jump

$$
\frac{\partial}{\partial \xi} \mathcal{G}_{\lambda}\left(\eta, \eta^{-}\right)-\frac{\partial}{\partial \xi} \mathcal{G}_{\lambda}\left(\eta, \eta^{+}\right)=(0,1,-(\lambda+1 / \tau))^{T} .
$$

Proposition 4.1. For any $\tau>1 / 4$

$$
\begin{gathered}
\sup _{\xi \in \mathbb{R}}\left\|\frac{\partial^{k} G_{\lambda}(\cdot, \xi)}{\partial \eta^{k}}\right\|_{L_{\eta}^{1}}, \quad \sup _{\eta \in \mathbb{R}}\left\|\frac{\partial^{k} G_{\lambda}(\eta, \cdot)}{\partial \eta^{k}}\right\|_{L_{\xi}^{1}}, \\
\sup _{\xi \in \mathbb{R}}\left\|\frac{\partial^{k+1} G_{\lambda}(\cdot, \xi)}{\partial \xi \partial \eta^{k}}\right\|_{L_{\eta}^{1}} \text { and } \sup _{\eta \in \mathbb{R}}\left\|\frac{\partial^{k+1} G_{\lambda}(\eta, \cdot)}{\partial \xi \partial \eta^{k}}\right\|_{L_{\xi}^{1}},
\end{gathered}
$$

for $k=0,1$ and 2, are uniformly bounded in $\lambda \in\{\lambda \in C: \operatorname{Re}(\lambda)>0\}$.

Proof. First we observe that there exist constants $\beta>0$ large enough and $\bar{C}, C>0$, all bounded in $\lambda$ on compact sets of $\Omega^{* *}$ such that

$$
\left|\frac{\partial^{k} G_{\lambda}(\eta, \xi)}{\partial \eta^{k}}\right| \leq \bar{C} e^{-\beta|\eta-\xi|}, \quad\left|\frac{\partial^{k+1} G_{\lambda}(\eta, \xi)}{\partial \xi \partial \eta^{k}}\right| \leq \bar{C} e^{-\beta|\eta-\xi|} \quad k=0,1,2 .
$$

This proof follows from the construction of $G_{\lambda}$ and Proposition 2.6. This gives the uniform estimates on the supremum of the $L^{1}$-norms as above in any compact set 
of $\Omega^{* *}$. In particular they hold for $\lambda$ in a set of the form $\Omega^{* *} \cap B_{M}(0)$ for some $M>0$.

It is then enough to prove the estimates in a set $\Omega^{* *} \backslash B_{M}(0)$ with $M$ as in Theorem 3.3. The estimates in such a set for $M$ (or $M-\varepsilon$ ) large enough (but fixed) are a consequence of Theorem 3.3. First, we observe that for $M$ as in the theorem then in fact $\Phi_{+}^{u}(\eta, \xi)=\Phi_{-}^{u}(\eta, \xi)$ and $\Phi_{+}^{s}(\eta, \xi)=\Phi_{-}^{s}(\eta, \xi)$, henceforth we drop the subscripts + and - of the flows.

In terms of the elements of the partial flows we then have that

$$
\mathcal{G}_{\lambda}(\eta, \xi)=\left\{\begin{array}{rc}
-\left(\Phi_{13}^{s}(\eta, \xi), \Phi_{23}^{s}(\eta, \xi), \Phi_{33}^{s}(\eta, \xi)\right)^{T}, & \eta>\xi \\
\left(\Phi_{13}^{u}(\eta, \xi), \Phi_{23}^{u}(\eta, \xi), \Phi_{33}^{u}(\eta, \xi)\right)^{T}, & \eta<\xi
\end{array}\right.
$$

which by recalling that

$$
\mathcal{G}_{\lambda}(\eta, \xi)=\left(G_{\lambda}(\eta, \xi), \frac{\partial G_{\lambda}(\eta, \xi)}{\partial \eta}, \frac{\left.\partial^{2} G_{\lambda}(\eta, \xi)\right)}{\partial \eta^{2}}\right)^{T}
$$

gives the expressions for $\partial^{k} G_{\lambda}(\eta, \xi) / \partial \eta^{k}$ with $k=0,1,2$. Similarly, computing the coordinates of (4.5) we have and expression for the rest of the partial derivatives

$$
\begin{gathered}
\frac{\partial G_{\lambda}(\eta, \xi)}{\partial \xi}=\left\{\begin{array}{rr}
\Phi_{12}^{s}(\eta, \xi)-(\lambda+1 / \tau) \Phi_{13}^{s}(\eta, \xi), & \eta>\xi \\
-\Phi_{12}^{u}(\eta, \xi)+(\lambda+1 / \tau) \Phi_{13}^{u}(\eta, \xi), & \eta<\xi
\end{array}\right. \\
\frac{\partial^{2} G_{\lambda}(\eta, \xi)}{\partial \xi \partial \eta}=\left\{\begin{array}{rr}
\Phi_{22}^{s}(\eta, \xi)-(\lambda+1 / \tau) \Phi_{23}^{s}(\eta, \xi), & \eta>\xi \\
-\Phi_{22}^{u}(\eta, \xi)+(\lambda+1 / \tau) \Phi_{23}^{u}(\eta, \xi), & \eta<\xi
\end{array}\right. \\
\frac{\partial^{3} G_{\lambda}(\eta, \xi)}{\partial \xi \partial \eta^{2}}=\left\{\begin{array}{rr}
\Phi_{32}^{s}(\eta, \xi)-(\lambda+1 / \tau) \Phi_{33}^{s}(\eta, \xi), & \eta>\xi \\
-\Phi_{32}^{u}(\eta, \xi)+(\lambda+1 / \tau) \Phi_{33}^{u}(\eta, \xi), & \eta<\xi .
\end{array}\right.
\end{gathered}
$$

And the uniform estimates on the $L^{1}$-norms of these elements follow now directly from Theorem 3.3 (ii).

We are now in the position to conclude that the spectral bound is actually the type of the semigroup.

TheOREM 4.2. For $\tau>1 / 4$ fixed, if the eigenvalue $\lambda=0$ of provides the spectral bound of $L$ in $H^{2}(\mathbb{R})$, then $L$ satisfies the $S D G$ property and travelling wave solutions of (1.1) with $\phi(-\infty)=0$ and $\phi(+\infty)=1$ are linearly stable.

Proof. By Prüss [23] we only need to get an estimate of the form

$$
\left\|(L-\lambda I)^{-1} f\right\|_{H^{2}} \leq C\|f\|_{H^{2}}
$$

with a $C>0$ independent of $\lambda$. We use the representation (4.1) and the inequality (1.10). The estimate on $L^{2}$ follows easily: for $f \in H^{2}(\mathbb{R})$ we have

$$
\left\|(L-\lambda I)^{-1} f\right\|_{L^{2}}=\left\|\int_{\mathbb{R}} G_{\lambda}(\cdot, \xi)\left(f(\xi)-\tau f^{\prime \prime}(\xi)\right) d \xi\right\|_{L^{2}} \leq C_{1}\left(\|f\|_{L^{2}}+\tau\left\|f^{\prime \prime}\right\|_{L^{2}}\right),
$$

where

$$
C_{1}:=\left(\sup _{\eta \in \mathbb{R}}\left\|G_{\lambda}(\eta, \cdot)\right\|_{L^{1}}\right)^{\frac{1}{2}}\left(\sup _{\xi \in \mathbb{R}}\left\|G_{\lambda}(\cdot, \xi)\right\|_{L^{1}}\right)^{\frac{1}{2}}
$$

is uniformly bounded in $\lambda$ by Proposition 4.1. To get an estimate on $\| \partial((L-$ $\left.\lambda I)^{-1} f\right) / \partial \eta \|_{L^{2}}$, we first write

$$
\frac{\partial}{\partial \eta} \int_{\mathbb{R}} G_{\lambda}(\eta, \xi)\left(f(\xi)-\tau f^{\prime \prime}(\xi)\right) d \xi=\int_{\mathbb{R}}\left(\frac{\partial G_{\lambda}(\eta, \xi)}{\partial \eta} f(\xi)+\tau \frac{\partial^{2} G_{\lambda}(\eta, \xi)}{\partial \eta \partial \xi} f^{\prime}(\xi)\right) d \xi
$$


Where we have used the first coordinate of the jump (4.6) and that for a function in $H^{2}$ there is $C^{1}$ function that coincides almost everywhere with it, and so the boundary terms at $\eta^{+}$and $\eta^{-}$cancel out. We now proceed as above, applying the estimate (1.10) and Proposition 4.1, and finally get that there is a constant $C_{2}>0$ independent of $\lambda$ such that

$$
\left\|\partial\left((L-\lambda I)^{-1} f\right) / \partial \eta\right\|_{L^{2}} \leq C_{2}\|f\|_{H^{1}} .
$$

It remains to get a similar estimate for $\left\|\partial^{2}\left((L-\lambda I)^{-1} f\right) / \partial \eta^{2}\right\|_{L^{2}}$. For $f \in H^{2}$ then

$$
\begin{array}{r}
\frac{\partial^{2}}{\partial \eta^{2}} \int_{\mathbb{R}} G_{\lambda}(\eta, \xi)\left(f(\xi)-\tau f^{\prime \prime}(\xi)\right) d \xi=\int_{\mathbb{R}} \frac{\partial^{2} G_{\lambda}(\eta, \xi)}{\partial \eta^{2}} f(\xi) d \xi \\
+\tau \int_{-\infty}^{\eta} \frac{\partial^{3} G_{\lambda}(\eta, \xi)}{\partial \eta^{2} \partial \xi} f^{\prime}(\xi) d \xi+\tau \int_{-\infty}^{\eta} \frac{\partial^{3} G_{\lambda}(\eta, \xi)}{\partial \eta^{2} \partial \xi} f^{\prime}(\xi) d \xi+2 \tau f^{\prime}(\eta),
\end{array}
$$

where we have used (4.6) (second coordinate). The expression is to be understood in a weak sense and is easily deduced from the weak formulation of the derivative. The $L^{2}$-norm of the above expression can then be bounded as before to yield

$$
\left\|\partial^{2}\left((L-\lambda I)^{-1} f\right) / \partial \eta^{2}\right\|_{L^{2}} \leq C_{3}\|f\|_{H^{1}}
$$

where $C_{3}$ is again independent of $\lambda$. Clearly, (4.8), (4.9) and (4.10) imply (4.7).

\section{Remarks on asymptotic stability on weighted spaces}

We end the section by addressing whether asymptotic (orbital) stability could be achieved in an exponentially weighted space. We introduce a weight function to $H^{2}(\mathbb{R})$, say $w \in C^{\infty}(\mathbb{R})$, with the properties

$$
\frac{d w}{d \eta}=a(\eta) w \quad \text { where } \lim _{\eta \rightarrow \pm \infty} a(\eta)=a^{ \pm}<\infty .
$$

The weighted space $H_{w}^{2}(\mathbb{R})$ is defined by

$$
H_{w}^{2}(\mathbb{R})=\left\{f: \frac{f}{w} \in H^{2}(\mathbb{R})\right\} .
$$

Next we derive conditions on $a(\eta)$ that ensure that the essential spectrum of $L$ in $H_{w}^{2}(\mathbb{R})$ is contained in the left half plane leaving $\lambda=0$ as an isolated eigenvalue. Thus, in particular, we require that $\phi^{\prime} \in H_{w}^{2}(\mathbb{R})$, i.e. that

$$
-\alpha^{+}-a^{+}<0 \text { and } \alpha^{-}-a^{-}>0 \text {. }
$$

That the point spectrum of $L$ as an operator in $H_{w}^{2}((\mathbb{R})$ is contained in the point spectrum of $L$ as an operator in $H^{2}(\mathbb{R})$ will be guaranteed by requiring that

$$
a^{+}<0 \text { and } a^{-}>0
$$

(as we shall see, if $V$ is an eigenfunction of $L$ in $H_{w}^{2}$ then $V \rightarrow 0$ exponentially as $|\eta| \rightarrow \infty$, and the above conditions imply that $U=w V \rightarrow 0$ as $|\eta| \rightarrow \infty$ too).

Before we analyse the essential spectrum, we need to clarify when the eigenvalue problem in $H_{w}^{2}$ can be reduced to studying a system of ODEs, as we did earlier in the $H^{2}$ setting. That $\lambda \in \rho(L)$ in $H_{w}^{2}$ is equivalent, by transforming with $V=U / w$, to the existence of a unique solution $V \in H^{2}$ of

$$
\frac{1}{w} L(w V)-\lambda V=g
$$


for any given $g \in H^{2}$. Recalling that $L$ is not a differential operator we write (5.4) in the more convenient form

$$
\frac{1}{w} \tilde{L}(w V)-\frac{1}{w}\left(I-\tau \frac{\partial^{2}}{\partial \eta^{2}}\right)(w V)=\tilde{g}
$$

with

$$
\tilde{g}=\frac{1}{w}\left(I-\tau \frac{\partial^{2}}{\partial \eta^{2}}\right)(w g)=: R_{w} g .
$$

And so solving (5.4) with $g \in H^{2}$ is equivalent to solving (5.5) for a given $\tilde{g} \in L^{2}$, provided the inverse of the operator $R_{w}$ is continuous from $L^{2}$ to $H^{2}$. The operator $R_{w}$ is given by

$$
R_{w} g=\left(1-\tau\left(a^{\prime}(\eta)+a(\eta)^{2}\right)\right) g-2 \tau a(\eta) \frac{d g}{d \eta}-\tau \frac{d^{2} g}{d \eta^{2}},
$$

and is invertible if for some $\varepsilon>0$

$$
1-\tau a^{2}(\eta)>\varepsilon \text { for all } \eta \in \mathbb{R},
$$

since this condition implies that the associated bilinear form is coercive in $H^{1}(\mathbb{R})$, cf. [3].

Summarising if $a$ satisfies (5.2), (5.3) and (5.7), we can study the essential spectrum by considering the equation (5.5), with $\tilde{g}$ set to 0 , as a system of linear ODEs, moreover $\lambda=0$ is an eigenvalue and the rest of the point spectrum of $L$ in the weighted space is contained in the point spectrum of $L$ considered in $H^{2}$.

As before the elements of the coefficient matrix tend to constant values as $\eta \rightarrow$ $-\infty$ and as $\eta \rightarrow+\infty$. The essential spectrum is the complement in $\mathbb{C}$ of the regions of consistent splitting for the associated limiting characteristic polynomials, that we denote by $P_{w}^{+}$and $P_{w}^{-}$, respectively. These are associated to the characteristic polynomials of $(2.6), P^{+}$and $P^{-}$, by

$$
P_{w}^{+}(\mu)=P^{+}\left(\mu+a^{+}\right), \quad P_{w}^{-}(\mu)=P^{-}\left(\mu+a^{-}\right)
$$

as the reader can check. Hence the sets where the roots of $P_{w}^{ \pm}$are purely imaginary are the curves in the complex plane

$$
S_{w}^{ \pm}=\left\{\lambda \in \mathbb{C}: \lambda=\frac{\tau\left(a^{ \pm}+i s\right)^{3}+\left(a^{ \pm}+i s\right)^{2} \pm\left(a^{ \pm}+i s\right)}{1-\tau\left(a^{ \pm}+i s\right)^{2}}, s \in \mathbb{R}\right\},
$$

and they enclose the essential spectrum. We only need to see if $a$ can be chosen such that for all $\lambda \in S_{w}^{+}$and for all $\lambda \in S_{w}^{-}$then $\operatorname{Re}(\lambda)<0$. It turns out that the conditions (5.2), (5.3) and (5.7) are sufficient.

For $\lambda \in S_{w}^{+}$we have

$$
\begin{aligned}
& \operatorname{Re}(\lambda)= \\
& \frac{\left(\tau^{2} a^{+}+\tau\right) s^{4}+\left(2 \tau^{2}\left(a^{+}\right)^{3}+2 a^{+}\left(2+a^{+}\right) \tau+1\right) s^{2}+\left(\tau^{2}\left(a^{+}\right)^{5}+\left(a^{+}\right)^{4} \tau-a^{+}\left(a^{+}+1\right)\right)}{\tau^{2} s^{4}+\left(2 \tau^{2}\left(a^{+}\right)^{2}+2 \tau\right) s^{2}+\left(1-\tau\left(a^{+}\right)^{2}\right)^{2}}
\end{aligned}
$$

which is strictly negative if the numerator of the expression is strictly negative. The numerator has been conveniently written as a polynomial in $s$. This can be solved for $s^{2}$. The coefficient of $s^{4}$ is positive by (5.2) (for $\tau>\frac{1}{4} \alpha^{+}=-1 / 2 \tau$ and $-1 / 2 \tau>-1 / \tau)$, so if

(5.8)

$\Delta=\left(2 \tau^{2}\left(a^{+}\right)^{3}+4 a^{+} \tau+2\left(a^{+}\right)^{2} \tau+1\right)^{2}-4\left(\tau^{2} a^{+}+\tau\left(\tau^{2}\left(a^{+}\right)^{5}+\left(a^{+}\right)^{4} \tau-a^{+}\left(1+a^{+}\right)\right)\right)<0$ 
then $\operatorname{Re}(\lambda)<0$. But $\Delta=16 \tau^{3}\left(a^{+}\right)^{4}+\tau(20 \tau+8)\left(a^{+}\right)^{2}+1+12 \tau a^{+}+24 \tau^{2}\left(a^{+}\right)^{3}$, where the last two terms are negative by (5.3). (5.2) and (5.7) imply in particular that $\left(a^{+}\right)^{2} \tau^{2}<1 / 4$ and $\left(a^{+}\right)^{2} \tau<1$ so

$$
\Delta<18+12 \tau a^{+}+24 \tau^{2}\left(a^{+}\right)^{3}<0
$$

where the last inequality follows easily by looking at the middle expression as a polynomial in $\tau$.

For $\lambda \in S_{w}^{-}$we have

$$
\operatorname{Re}(\lambda)=-\frac{A s^{4}+B s^{2}+C}{\tau^{2} s^{4}+\left(2 \tau^{2}\left(a^{+}\right)^{2}+2 \tau\right) s^{2}+\left(1-\tau\left(a^{+}\right)^{2}\right)^{2}}
$$

with

$$
\begin{aligned}
& A=\tau^{2} a^{-}+\tau \\
& B=2 \tau^{2}\left(a^{-}\right)^{3}+2 a^{-} \tau+2\left(a^{-}\right)^{2} \tau+1 \\
& C=\left(a^{-}\right)^{5} \tau^{2}+\left(a^{-}-2\right)\left(a^{-}\right)^{3} \tau+a^{-}\left(1-a^{-}\right)
\end{aligned}
$$

The discriminant of the numerator has

$$
\Delta=B^{2}-4 A C=16 \tau^{3}\left(a^{-}\right)^{4}+24 \tau^{2}\left(a^{-}\right)^{3}+8 \tau\left(a^{-}\right)^{2}+1>0 .
$$

However, $A>0$ and $B>0$, and (5.2) (recalling that $\alpha^{-}=(\sqrt{1+4 \tau}-1) / 2 \tau$ ) and (5.3) imply that $C>0$, and so the numerator does not change sign with $s \in \mathbb{R}$, finally giving that $\operatorname{Re}(\lambda)<0$ (using again $A>0$ ).

We have proved the following result.

THEOREM 5.1. Let $\tau>1 / 4$ be fixed and let $w$ be a weight function satisfying (5.2), (5.3) and (5.7). Then if the eigenvalue $\lambda=0$ provides the spectral bound of $L$ in $H^{2}(\mathbb{R})$, it does too for $L$ considered in $H_{w}^{2}$ and moreover $\lambda=0$ is isolated. Thus provided that the SDG property holds in $H_{w}^{2}$, travelling wave solutions of (1.1) with $\phi(-\infty)=0$ and $\phi(+\infty)=1$ are linearly (orbitally) asymptotically stable in $H_{w}^{2}(\mathbb{R})$.

To proof that the SDG property holds in $H_{w}^{2}$ might be achieved by pursuing the resolvent estimates for the problem (5.4). We shall not do this here, but remark that it is natural to expect to get uniform estimates on the resolvent operator too. The key estimates for large $|\lambda|$ would not change significantly; the eigenvalues of the matrix coefficient of the corresponding system of ODEs are essentially shifted by $a(\eta)$ which is real and independent of $\lambda$.

\section{Large $\tau$ : numerical search for zeros}

The aim of this section is to provide numerical evidence of linear stability of travelling wave solutions for large values of $\tau$ by evaluting the Evans function in a wide contour. We indicate the dependence on $\tau$ of $D(\lambda)$ by a subindex, $D_{\tau}$, below. The numerical results are performed by using the fact that the zeros of $D_{\tau}(\lambda)$ can only emerge in pairs through the imaginary axis as $\tau$ increases:

Proposition 6.1. Suppose that there exists $\tau^{*}>\frac{1}{4}$ such that $D_{\tau^{*}}(\lambda)$ has a zero at some $\lambda^{*}$ with positive real part. Then there exists $\bar{\tau} \in\left(\frac{1}{4}, \tau^{*}\right]$ such that $D_{\bar{\tau}}(\lambda)$ has a pair of zeros in the imaginary axis aside from the origin. 
ProOF. It is a consequence of the continuity of $D_{\tau}(\lambda)$ in $\tau$, (3.9) (Theorem 3.3(i)) and Rouche's theorem. Rouche's theorem says that if two analytic function are close to each other in a simply connected domain, then they will have the same number of zeros (counting multiplicity) in that domain; since for $\tau \leq \frac{1}{4}$ there are no eigenvalues with positive real part in the right hand plane, the only way zeros can enter this domain as $\tau$ increases is if they pop up from $\infty$ or cross the imaginary axis from the left half plane. The first possibility contradicts (3.9). Thus for $D_{\tau^{*}}(\lambda)$ to have a zero in the right half plane, there must exist $\bar{\tau}<\tau^{*}$ and $\bar{\lambda}$ with $\operatorname{Re}(\bar{\lambda})=0$ such that $D_{\bar{\tau}}(\bar{\lambda})=0 . \bar{\lambda} \neq 0$ since by Lemma $2.10, D_{\tau}^{\prime}(0) \neq 0$ for all $\tau$. Then $\bar{\lambda}=s i$ for some $s \in \mathbb{R} \backslash\{0\}$, and Lemma 2.8-(iii) implies that $D_{\bar{\tau}}(-s i)=0$ as well.

We compute numerically the Evans function along the imaginary axis. Since by (3.9), zeros of $D_{\tau}(\lambda)$ only enter the right half plane through the imaginary axis, we take the imaginary axis as a wide contour around the right half plane. We look at the number of times that the graph of the curve $s \in \mathbb{R} \rightarrow D_{\tau}(s i)$ wraps around the origin (Winding number), this gives the number of zeros of $D_{\tau}(\lambda)$ in the right half plane, and hence, the number of isolated eigenvalues of the operator $L$.

In Figure 2 we have plotted the graphs of $D_{\tau}(\lambda)$ along the imaginary axis, for several values of $\tau$, including $\tau=0$. As a guideline to interpret the results notice that if a new zero of $D_{\tau}(\lambda)$ appears through the imaginary axis, the curve $D_{\tau}(s i)$ must intersect itself at the origin (since $D_{\tau}(i s)=0$ for $s=0$ and for some $s=\tilde{s}$ and $s-\tilde{s})$. However, the numerical results do not exhibit these self-intersections. And the evolution of the graphs with respect to $\tau$ suggests that this is not going to happen at very large values of $\tau$. $Y_{1}^{-}$by

We have approximated the Evans function at each $\lambda$-value by first transforming

where

$$
\mathcal{V}^{-}(\eta, \lambda)=\exp \left(-\mu_{1}(\eta, \lambda) \eta\right) Y_{1}^{-}(\eta, \lambda)
$$

$$
\mu_{1}(\eta, \lambda)= \begin{cases}\mu_{1}^{-}(\lambda) & \text { if } \eta<\eta_{m} \\ \mu_{1}^{+}(\lambda) & \text { if } \eta \geq \eta_{m}\end{cases}
$$

for some $\eta_{m} \in \mathbb{R}$. Now $\mathcal{V}^{-}$satisfies

$$
\begin{array}{r}
\mathcal{V}^{-}(\eta, \lambda) \sim v_{-}(\lambda) \quad \text { as } \eta \rightarrow-\infty \\
\mathcal{V}^{-}(\eta, \lambda) \sim v_{+}(\lambda) D_{\tau}(\lambda) \quad \text { as } \eta \rightarrow+\infty
\end{array}
$$

and the equation

$$
\frac{d \mathcal{V}^{-}(\eta, \lambda)}{d \eta}=-\mu_{1}(\eta, \lambda) \mathcal{V}^{-}(\eta, \lambda)+A(\lambda, \eta) \mathcal{V}^{-}(\eta, \lambda)
$$

We approximate numerically this equation on a finite interval $\left[\eta_{0}, \eta_{f}\right]$. First on the interval $\left[\eta_{0}, \eta_{m}\right]$ we solve the equation

$$
\frac{d \mathcal{V}^{-}}{d \eta}=-\mu_{1}^{-}(\lambda) \mathcal{V}^{-}+A(\lambda, \eta) \mathcal{V}^{-}
$$

for $\eta_{0}<0$ sufficiently small, with initial condition the eigenvector $\left(1, \mu_{1}^{-},\left(\mu_{1}^{-}\right)^{2}\right)$. On the interval $\left[\eta_{m}, \eta_{f}\right]$ we solve

$$
\frac{d \mathcal{V}^{-}}{d \eta}=-\mu_{1}^{+}(\lambda) \mathcal{V}^{-}+A(\lambda, \eta) \mathcal{V}^{-}
$$


for $\eta_{f}>0$ sufficiently large. The initial condition at $\eta_{m}$ is taken to be the value of $\mathcal{V}^{-}$at $\eta_{m}$ obtained after solving (6.1). We capture the value of $\mathcal{V}^{-}$at $\eta_{f}$ and use it to approximate $D_{\tau}(\lambda)$. We then take

$$
D_{\tau}(\lambda)=w^{+} \mathcal{V}^{-}\left(\eta_{f}\right)
$$

In practise this computation is repeated at each value $\lambda=s i$, where $s \in[-100,100]$. We have only discretised the interval $[0,100]$, since the symmetry of $D_{\tau}$ gives $D_{\tau}(-s i)$ as $\overline{D_{\tau}(s i)}$. The partition of the $s$-interval is not uniform. We use a finer grid near 0 . From 0 to 1 we take 0.001 as $s$-step size, and 0.5 for the rest of the interval. At each $s$-step the systems (6.1) and (6.2) are solved simultaneously with the travelling wave equation (2.1). Here we have taken $\eta_{0}=-50, \eta_{m}=0$ and $\eta_{f}=500$. We have used the Runge-Kutta solver implemented for Matlab ode45.

\section{Appendix}

A.1. Exponential dichotomies and linear ODEs. This section revises some of the material exposed in [25] and proved in [5], [18] and [19]. Much of the ideas in the proofs are standard in the theory of stability of linear ODE and of their perturbations. We recall also a result that appears in [4] with regard to the proof of Proposition 2.5.

We start with the definition of exponential dichotomies for a general linear system of ODEs and give the fundamental result regarding small perturbations. We shall rephrase these in a way that can be immediately applied to the current problem.

Let $A$ be a $n \times n$ matrix and consider the linear system of ODE's

$$
\frac{d}{d \eta} Y(\eta)=A(\eta) Y(\eta)
$$

on a domain $I \subset \mathbb{R}$ containing $\eta=0$. Let $\Phi(\eta)$ denote a matrix of fundamental solutions of (A.1) with $\Phi(0)=I$, and $\Phi(\eta, \xi)$ the flow induced by $A$, i.e. $\Phi(\eta, \xi)=$ $\Phi(\eta) \Phi^{-1}(\xi)$.

Definition A.2. The system (A.1) has exponential dichotomies in a domain $I$, where $I=\mathbb{R}^{+}, \mathbb{R}^{-}$or $\mathbb{R}$ if there exists a family of projections $\mathcal{Q}(\eta)$ continuous in $\eta$, and constants $\delta \geq 0, \kappa_{s}, \kappa_{u}$ and $K$ with $\kappa_{s}<\delta<\kappa_{u}, K>0$ such that

(1) letting $\Phi^{s}(\eta, \xi):=\Phi(\eta, \xi) \mathcal{Q}(\xi)$ and $\Phi^{u}(\eta, \xi):=\Phi(\eta, \xi)(I-\mathcal{Q}(\xi))$ be the partial flows associated to $\mathcal{Q}$, then for every $\eta, \xi \in I$

$$
\begin{aligned}
& \left|\Phi^{s}(\eta, \xi)\right| \leq K e^{\kappa_{s}(\eta-\xi)}, \quad \eta \geq \xi, \\
& \left|\Phi^{u}(\eta, \xi)\right| \leq K e^{\kappa_{u}(\eta-\xi)}, \quad \eta \leq \xi .
\end{aligned}
$$

(2) the projections commute with the flows, i.e. $\Phi(\eta, \xi) \mathcal{Q}(\xi)=\mathcal{Q}(\eta) \Phi(\eta, \xi)$, and for every $\eta, \xi \in I$

$$
\begin{array}{lll}
\Phi^{s}(\eta, \xi) Y_{0} & \in \mathrm{R}(\mathcal{Q}(\eta)), & \eta \geq \xi \\
\Phi^{u}(\eta, \xi) Y_{0} & \in \mathrm{N}(\mathcal{Q}(\eta)), & \eta \leq \xi .
\end{array}
$$

Also $\operatorname{dim} N(\mathcal{Q}(\eta))$ is independent of $\eta$, and is referred to as the Morse index of the exponential dichotomy.

Observe that when $\delta=0$, then $\Phi^{s}(\eta, \xi)$ gives solutions to (A.1) that decay exponentially for $\eta \geq \xi$, and $\Phi^{u}(\eta, \xi)$ gives the solutions that decay, in backward direction, exponentially for $\eta \leq \xi$. 


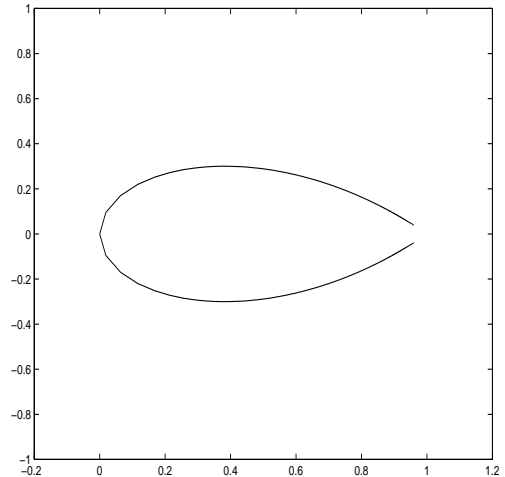

(a) $\tau=0$

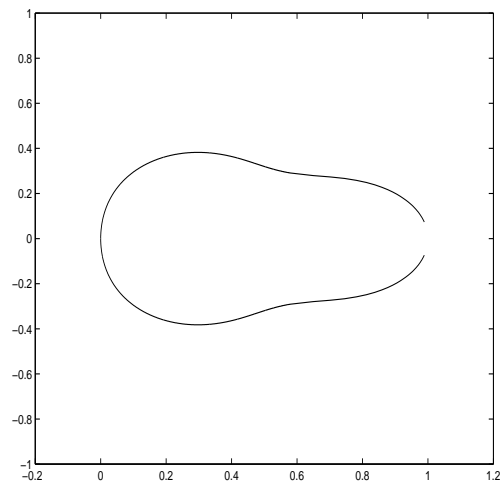

(c) $\tau=1$

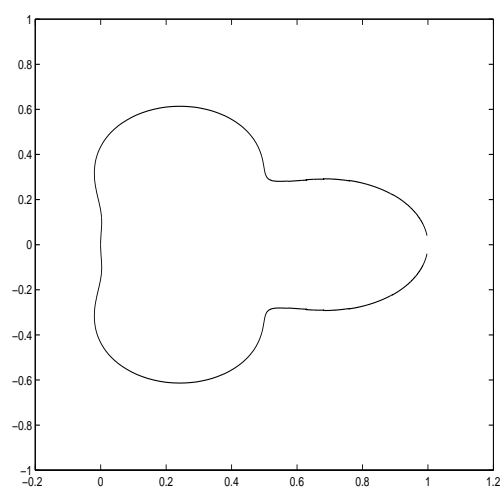

(e) $\tau=10$

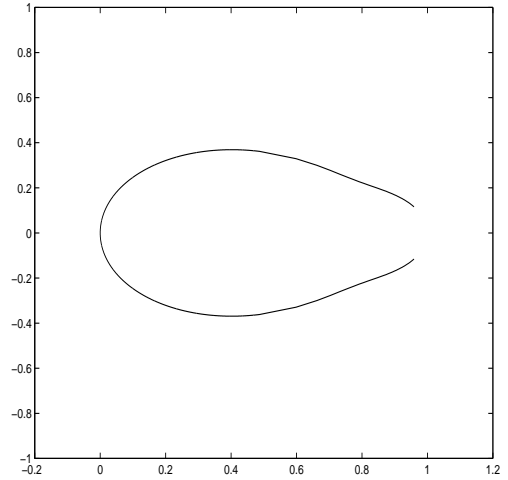

(b) $\tau=0.2$

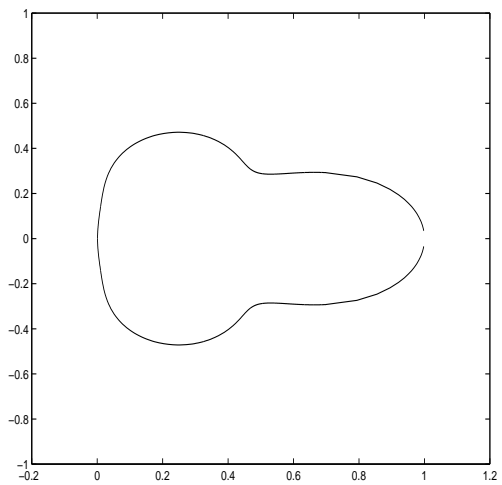

(d) $\tau=5$

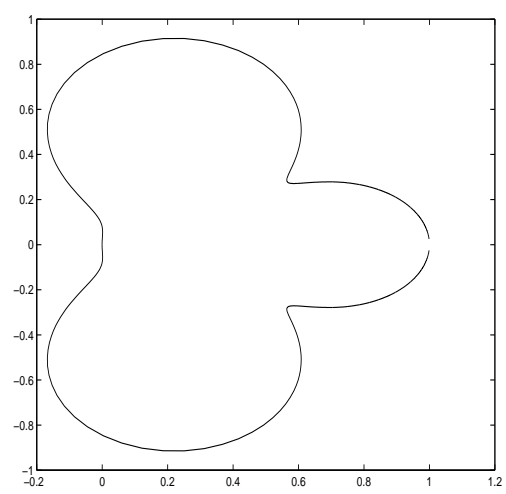

(f) $\tau=20$

Figure 2. $\left\{D_{\tau}(s i): s \in \mathbb{R}\right\} \subset \mathbb{C}$ for different values of $\tau$.

In what follows we shall indicate with a subindex + that the dichotomy is defined on $[0, \infty)$ and with a subindex - that it is defined on $(-\infty, 0]$.

Exponential dichotomies persist under small perturbations of (A.1) (the roughness theorem), a result that we will refer to in sections 2 and 3 . For the later the 
following form of the theorem will be used. The form used for the former version is explained below.

THEOREM A.3 (The roughness theorem, $[5]$ ). Let $I=\mathbb{R}$ and suppose that $A(\eta)$ is smooth, and that (A.1) has an exponential dichotomy satisfying Definition A.2. Let $B(\eta)$ be a continuous and bounded matrix and let $\varepsilon>0$. Then there exist a small enough constant $\varepsilon^{*}$ and a constant $\tilde{K}>0$ such that the equation

$$
\frac{d}{d \eta} Y(\eta)=(A(\eta)+\varepsilon B(\eta)) Y(\eta)
$$

has an exponential dichotomy on $\mathbb{R}$ for all $\varepsilon<\varepsilon^{*}$ with the constants in (i) of Definition A.2 replaced by $\tilde{K}, \kappa_{s}+\varepsilon$ and $\kappa_{u}-\varepsilon$. Moreover, the projections $\mathcal{P}(\xi)$ and flows $\Phi^{2}(\eta, \xi), \Phi(\eta, \xi)$ associated to (A.2) are $\varepsilon$-close to those associated to (A.1) for all $\eta, \xi$ in $\mathbb{R}$.

For details of the proof with (A.5) replaced by a small term (either linear or nonlinear) see [5]. For the general result see [18] (the statement in general requires that the matrix $A$, of any finite dimension, is hyperbolic). For similar results in abstract evolution problems where $A$ is a sectorial operator on a Banach space, see [24] with a smallness assumption on $B$ and [22] with a compactness assumption on $B$. We give the main ideas below.

Let us now assume that $A$ has constant coefficients, that $n=3$ and that the characteristic roots of $A, \mu_{1}, \mu_{2}$ and $\mu_{3}$, satisfy

$$
\operatorname{Re}\left(\mu_{1}\right)>0>\max \left\{\operatorname{Re}\left(\mu_{2}\right), \operatorname{Re}\left(\mu_{3}\right)\right\},
$$

where we do not exclude the possibilities that $\mu_{2}=\mu_{3}$ or $\operatorname{Re}\left(\mu_{2}\right)=\operatorname{Re}\left(\mu_{3}\right)$. Let us now consider a perturbation of (A.1), namely

$$
\frac{d}{d \eta} Y(\eta)=(A+B(\eta)) Y(\eta)
$$

where $B(\eta)$ is a matrix with smooth coefficients and such that there are positive constants $\beta$ and $C$ with

$$
|B(\eta)| \leq C e^{-\beta|\eta|}
$$

Let $\mathcal{P}_{1}$ be the spectral projection associated to the eigenvalue $\mu_{1}$, let also $\mathcal{Q}_{1}:=$ $I-\mathcal{P}_{1}$. Letting $E^{u}:=R\left(\mathcal{P}_{1}\right)$ denote the eigenspace associated to $\mu_{1}$, let $v_{1}$ be an eigenvector generating this eigenspace. Then $E^{s}:=R\left(\mathcal{Q}_{1}\right)\left(=N\left(\mathcal{P}_{1}\right)\right)$ is the eigenspace associated to $\mu_{2}$ and $\mu_{3}$ and let $v_{2}$ and $v_{3}$ be left eigenvectors that span $E^{s}$, which are the eigenvectors associated to $\mu_{2}$ and $\mu_{3}$ respectively when $\mu_{2} \neq \mu_{3}$.

Then the following classical result hold.

Theorem A.4 (Adapted from [4], Theorem 11). If $A$ has three distinct eigenvalues and under the assumptions (A.3) and (A.5), there exist solutions of $(A .4)$ and positive constants $C_{-}^{i}$ and $C_{+}^{i}$ for $i=1,2,3$ with

$$
\begin{array}{cc}
\left|Y_{-}^{i}(\eta)-v_{i} e^{\mu_{i} \eta}\right| \leq C_{-}^{i} e^{+\beta \eta} \quad \eta \leq 0, \\
\left|Y_{+}^{i}(\eta)-v_{i} e^{\mu_{i} \eta}\right| \leq C_{+}^{i} e^{-\beta \eta} \quad \eta \geq 0,
\end{array}
$$

that are uniquely determined and linearly independent for all $i=1,2,3$.

The result, in fact, holds for a smooth matrix $A(\eta)$ that approaches constant matrices as $\eta \rightarrow \infty$ and as $\eta \rightarrow-\infty$, and has simple eigenvalues $\mu_{i}(\eta)$ for all $\eta$ that tend to the simple eigenvalues of the limiting matrices. The proof uses a projection 
into the direct sum of eigenspaces of $A-\mu_{i}$ such that the eigenvalues $\mu_{j}$ with $j \neq i$ satisfy that the sign of $\operatorname{Re}\left(\mu_{j}(\eta)-\mu_{i}(\eta)\right)$ does not change with $\eta \in I$. The argument does not apply if $\mu_{2}=\mu_{3}$, but that does not exclude the possibility that $\operatorname{Re}\left(\mu_{i}(\eta)\right) \equiv \operatorname{Re}\left(\mu_{j}(\eta)\right)$ for all $\eta$. This last point seems to have been overlooked in [17]. So in the present case it might be that $\operatorname{Re}\left(\mu_{2}\right)=\operatorname{Re}\left(\mu_{3}\right)$.

If $A$ depends analytically on a parameter $\lambda \in \mathbb{C}, A=A(\eta, \lambda)$, and there is a domain $\Omega \subset \mathbb{C}$ such that for all $\lambda \in \Omega$ the characteristic roots of $A$ satisfy (A.3) then Theorem A.4 holds true locally analytically in $\lambda$ (note that all constants depend now on $\lambda$ ).

Clearly, the spectral projections $\mathcal{P}_{1}$ and $\mathcal{Q}_{1}$ give an exponential dichotomy for (A.1), with partial flows

$$
\Phi^{s}\left(\eta, \eta_{0}\right):=\Phi\left(\eta, \eta_{0}\right) \mathcal{Q}_{1}, \quad \Phi^{u}\left(\eta, \eta_{0}\right):=\Phi\left(\eta, \eta_{0}\right) \mathcal{P}_{1}, \quad \text { for all } \quad \eta, \eta_{0} \in \mathbb{R},
$$

and it satisfies (ii) of Definition A.2 with

$$
\kappa_{s}=\min \left\{\operatorname{Re}\left(\mu_{2}\right), \operatorname{Re}\left(\mu_{3}\right)\right\}<0, \quad \kappa_{u}=\operatorname{Re}\left(\mu_{1}\right)>0 .
$$

THEOREM A.5 ([18]). Under the assumptions (A.3) and (A.5), there is an exponential dichotomy with flows $\tilde{\Phi}_{+}^{s}(\eta, \xi)$ and $\tilde{\Phi}_{+}^{u}(\eta, \xi)$ for all $\eta, \xi \in \mathbb{R}^{+}$associated to (A.4) with projection

$$
\mathcal{Q}_{+}(\xi):=\tilde{\Phi}_{+}^{s}(\xi, \xi) .
$$

Moreover, its Morse index is 1 (it coincides with the Morse index of the dichotomy of (A.1) i.e. with dim $\left.E^{u}\right)$. The exponential estimates are as follows: If $\varepsilon>0$ is small enough and $L>0$ is large enough so that $\varepsilon<\kappa_{u}$ and $\sup _{|\eta|<L}|B(\eta)|<C \varepsilon$, then (i) of Definition A.2 holds with $\kappa_{u}$ replaced by $\kappa_{u}-\varepsilon$ and $\kappa_{s}$ replaced by $\kappa_{s}+\varepsilon$ for $\xi, \eta$ in $(-\infty, L]$. The analogous statement holds for the system considered on $\mathbb{R}^{-}$.

The crucial point of the proof of the roughness theorems is the following representation of the dichotomies, for example for $\eta$ and $\xi$ in $(-\infty, 0]$

$$
\begin{array}{r}
\tilde{\Phi}_{-}^{s}(\eta, \xi)=\Phi_{-}^{s}(\eta, \xi)-\int_{\eta}^{0} \Phi_{-}^{u}(\eta, s) B(s) \tilde{\Phi}_{-}^{s}(s, \xi) d s+\int_{\xi}^{\eta} \Phi_{-}^{s}(\eta, s) B(s) \tilde{\Phi}_{-}^{s}(s, \xi) d s \\
(\mathrm{~A} .9) \quad-\int_{-\infty}^{\xi} \Phi_{-}^{s}(\eta, s) B(s) \tilde{\Phi}_{-}^{u}(s, \xi) d s \quad \text { for } \quad \xi \leq \eta \leq 0, \\
\tilde{\Phi}_{-}^{u}(\eta, \xi)=\Phi_{-}^{u}(\eta, \xi)+\int_{\xi}^{\eta} \Phi_{-}^{u}(\eta, s) B(s) \tilde{\Phi}_{-}^{u}(s, \xi) d s+\int_{-\infty}^{\eta} \Phi_{-}^{s}(\eta, s) B(s) \tilde{\Phi}_{-}^{u}(s, \xi) d s \\
(\mathrm{~A} .10) \\
+\int_{\xi}^{0} \Phi_{-}^{u}(\eta, s) B(s) \tilde{\Phi}_{-}^{s}(s, \xi) d s \text { for } \eta \leq \xi \leq 0 .
\end{array}
$$

This defines a fixed-point map, that, by the properties of $B$, is a contraction on $L^{\infty}(-\infty, \bar{\eta})$ for a small enough $\bar{\eta}$. The fixed-point can be extended to the rest of the domain, by continuity of solutions, and the representation formula above and Gronwall's lemma provide the estimate in the rest of the domain. The representation (A.9)-(A.10) is motivated by the following observation. One can prove (see e.g. [4]) that bounded solutions, $Y_{1}$ say, on the interval $(-\infty, \xi]$ for any $\xi<0$ are 
given by the formula

$$
\begin{gathered}
Y_{1}(\eta)=\Phi_{-}^{u}(\eta, \xi) Y_{1}(\xi)+\int_{\xi}^{\eta} \Phi_{-}^{u}(\eta, s) B(s) Y_{1}(s) d s \\
+\int_{-\infty}^{\eta} \Phi_{-}^{s}(\eta, s) B(s) Y_{1}(s) d s \quad \text { with } \quad \eta<\xi .
\end{gathered}
$$

Let now a solution $Y_{2}(\eta)$ given on the interval $[\xi, 0]$ by

$$
\begin{aligned}
Y_{2}(\eta) & =\Phi_{-}^{s}(\eta, \xi) Y_{2}(\xi)+\int_{\xi}^{\eta} \Phi_{-}^{s}(\eta, s) B(s) Y_{2}(s) d s \\
& -\int_{\eta}^{0} \Phi_{-}^{u}(\eta, s) B(s) Y_{2}(s) d s \quad \text { with } \quad \eta \geq \xi .
\end{aligned}
$$

Then a function $Y(\eta)$ given by $Y(\eta)=Y_{1}(\eta)$ for $\eta \in(-\infty, \xi]$ and by $Y(\eta)=Y_{2}(\eta)$ for $\eta \in(\xi, 0]$ is a solution of (A.4) in $(-\infty, 0]$ with $Y(\xi)=Y_{1}(\xi)+Y_{2}(\xi)$.

The exponential dichotomies are related to bounded solutions as follows. Suppose first that (A.4) has exponential dichotomies given by (A.9)-(A.10), then straightforward differentiation shows that $\tilde{\Phi}_{-}(\eta, \xi)=\tilde{\Phi}_{-}^{s}(\eta, \xi)+\tilde{\Phi}_{-}^{u}(\eta, \xi)$ defines the flow of (A.4) on $(-\infty, 0]$. Conversely, if $Y_{1}$ is a bounded solution of (A.4) in $(-\infty, \xi]$ and $Y_{2}$ is a bounded solution of (A.4) in [ $\xi, 0]$ given by (A.12) and such that $Y_{1}(\xi) \neq Y_{2}(\xi)$, then taking $Y(\xi)=Y_{1}(\xi)+Y_{2}(\xi)$ it is easy to see that the pair $\left(Y_{1}, Y_{2}\right)$ satisfies (A.9)-(A.10) (applied to the initial condition $Y(\xi)$ ) with $\tilde{\Phi}_{-}^{s}(\eta, \xi) Y(\xi)$ replaced by $Y_{1}(\eta)$ and $\tilde{\Phi}_{-}^{u}(\eta, \xi) Y(\xi)$ replaced by $\left.Y_{2}(\eta)\right)$, and clearly the projection associated that defines $\Phi_{-}^{u}, \mathcal{P}_{-}$is determined by

$$
\Phi(\eta, \xi) \mathcal{P}_{-}(\xi) Y(\xi)=Y_{1}(\eta) \quad\left(\Leftrightarrow \quad \mathcal{P}_{-}(\xi) Y(\xi)=Y_{1}(\xi)\right) .
$$

Finally, we recall that if $A$ depends analytically or smoothly on a second parameter $\lambda \in \Omega$ such that (A.3) holds, then dichotomies can be chosen to depend analytically/continuously in $\lambda \in \Omega,([\mathbf{2 4}],[\mathbf{2 2}])$.

\section{References}

1. J. Alexander, R. Gardner, and C. Jones, A topological invariant arising in the stability analysis of travelling waves, J. Reine Angew. Math. 410 (1990), 167-212.

2. T. B. Benjamin, J. L. Bona, and J. J. Mahony, Model equations for long waves in nonlinear dispersive systems, Philos. Trans. Roy. Soc. London Ser. A 272 (1972), no. 1220, 47-78.

3. H. Brezis, Analyse fonctionnelle : theorie et applications, Masson, 1983.

4. W. A. Coppel, Stability and asymptotic behavior of differential equations, D. C. Heath and Co., Boston, Mass., 1965.

5. _ Dichotomies in stability theory, Lecture Notes in Mathematics, Vol. 629, SpringerVerlag, Berlin, 1978. MR MR0481196 (58 \#1332)

6. C. Cuesta and J. Hulshof, A model problem for groundwater flow with dynamic capillary pressure: stability of travelling waves, Nonlinear Anal. 52 (2003), 1199-1218.

7. C. Cuesta, C. J. van Duijn, and J. Hulshof, Infiltration in porous media with dynamic capillary pressure: travelling waves, European J. Appl. Math. 11 (2000), 381-397.

8. C. M. Cuesta, Pseudo-parabolic equations with driving convection term, Ph.D. thesis, Vrije Universiteit Amsterdam, 2003.

9. John W. Evans, Nerve axon equations. III. Stability of the nerve impulse, Indiana Univ. Math. J. $22(1972 / 73), 577-593$.

10. Robert A. Gardner and Kevin Zumbrun, The gap lemma and geometric criteria for instability of viscous shock profiles, Comm. Pure Appl. Math. 51 (1998), no. 7, 797-855.

11. S. M. Hassanizadeh and W. G. Gray, Thermodynamic basis of capillary pressure in porous media., Water Resour. Res. 29 (1993), 3389-3405. 
12. Daniel Henry, Geometric theory of semilinear parabolic equations, Springer-Verlag, Berlin, 1981.

13. Peter Howard, Pointwise Green's function approach to stability for scalar conservation laws, Comm. Pure Appl. Math. 52 (1999), 1295-1313.

14. Peter Howard and Kevin Zumbrun, Pointwise estimates and stability for dispersive-diffusive shock waves, Arch. Ration. Mech. Anal. 155 (2000), no. 2, 85-169.

15. Todd Kapitula, The Evans function and generalized Melnikov integrals, SIAM J. Math. Anal. 30 (1999), no. 2, 273-297 (electronic).

16. T. Kato, A spectral mapping theorem for the exponential function, and some counterexamples, Tech. report, University of Wisconsin-Madison, Mathematical research center, 1982.

17. Corrado Mascia and Kevin Zumbrun, Pointwise Green's function bounds and stability of relaxation shocks, Indiana Univ. Math. J. 51 (2002), 773-904.

18. Kenneth J. Palmer, Exponential dichotomies and transversal homoclinic points, J. Differential Equations 55 (1984), 225-256.

19. Exponential dichotomies and Fredholm operators, Proc. Amer. Math. Soc. 104 (1988), 149-156.

20. A. Pazy, Semigroups of linear operators and applications to partial differential equations, Springer-Verlag, New York, 1983.

21. Robert L. Pego and Michael I. Weinstein, Eigenvalues, and instabilities of solitary waves, Philos. Trans. Roy. Soc. London Ser. A 340 (1992), no. 1656, 47-94.

22. Daniela Peterhof, Björn Sandstede, and Arnd Scheel, Exponential dichotomies for solitarywave solutions of semilinear elliptic equations on infinite cylinders, J. Differential Equations 140 (1997), 266-308.

23. Jan Prüss, On the spectrum of $C_{0}$-semigroups, Trans. Amer. Math. Soc. 284 (1984), 847-857.

24. B. Sandstede, Verzweigungstheorie homokliner verdopplungen, Ph.D. thesis, Univeristy of Stuttgart, 1993.

25. Björn Sandstede, Stability of travelling waves, Handbook of dynamical systems, Vol. 2, NorthHolland, Amsterdam, 2002, pp. 983-1055.

26. D. H. Sattinger, Stability of travelling waves of nonlinear parabolic equations, VII. Internationale Konferenz über Nichtlineare Schwingungen (Berlin, 1975), Band I, Teil 2, AkademieVerlag, Berlin, 1977, pp. 209-213. Abh. Akad. Wissensch. DDR, Abt. Math.-Naturwissensch.Techn., No. $4 \mathrm{~N}$

27. Kevin Zumbrun and Peter Howard, Pointwise semigroup methods and stability of viscous shock waves, Indiana Univ. Math. J. 47 (1998), 741-871.

Instituto de Ciencias Matemáticas CSIC-UAM-UC3M-UCM, Facultad de Ciencias, Univ ersidad Autónoma de Madrid, Crta. Colmenar Viejo Km 15, 28049, Madrid, Spain. E-mail address: carlota.cuesta@icmat.es 